\title{
Étude expérimentale de l'écoulement turbulent dans un conduit divergent parcouru par de l'air
}

\author{
The experimental of turbulent air flow \\ in a divergent pipe
}

\author{
PAR .J.P. MILLIAT
}

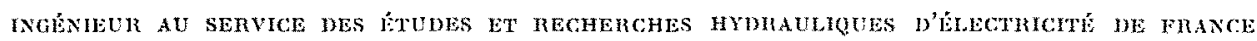

\begin{abstract}
Elude experimentale de l'écoulement turbulent dans un canal bi-dimensionnel constitué par un tronçon à parois parallèles prolongé par un troncon divergent d'angle total égal à 2, 4 et 6 degrés suivant les essais.

Mesures à l'anémomètre à fil chaud, duns differentes sections transuersales, des vitesses moyennes et des fluctuations de ces vitesses (moyenne quadratique, spectre, coefficients de corrélation).

Mise en évidence d'un régime d'śquilibre de l'éconlement dans la partie awal du divergent. après une zone de transition di l'amont; éfude des caractéristiques de ces regimes d'équilibre en fonction de l'angle de dinergence.

Représentation des fonctions spectrales des fluctuations de vitesse en coordonnes non di. mensionnelles. Existence d'une certaine intermittence (alternance du régime laminaire et du régime turbulent) dans l'écoulement an voisinage immédiat de la paroi.
\end{abstract}

\begin{abstract}
An experimental investigution of turbulent flow in a two dimensional channel consisting of a parallel walled section continued by a section with walls diverging at tolul angles of 2 , a and 6 degrees according to the tests.

Measurements made, on varions cross sections whith a hot wive anemometer, of abertge nelocities and of fucluations of these velocities fquadratic mean, spectum and correlation roefficients).

Revelation of a state of equitibrinm in the fow downstream of the dinerying section following a transition zone upstream. Investigation of the characteristics of these states of equilibrian in terms of the angle of divergence.

Representing spectrum functions of the velocity furctualions in lerms of non-dimenstonal coordinates. The existence of an infermittent state of flow in the immediate neighbonhood of the walls falternation betmeen laminar and lurbulent conditions).
\end{abstract}

\section{I. - INTRODUCTION}

Afin de mieux siluer le probleme particulier itudié dans cette note, nous rappellerons brièvement, sans prétendre en fairc un tableau complet, différentes recherches concernant le mécaaisme des écoulements turbulents et diverses ctapes vers la solution d'un problème dont beaucoup d'aspects nous sont encore cachés.

Une des premieres tentatives d'explication, née des études expérimentales enlreprises en aérodynamique a propos de la couche limite et en hydraulique avec l'écoulement dans les tuyaux, est basce sur la notion de longueur de mélange imagince par Prandrt. Si les hypotheses faites dans cette théorie permetlent généralement de prévoir des distributions de vitesses moyennes vérifiant bien les courbes expérimentales, elles ne donnent par contre aucun renseignement sur le champ des vilesses turbulentes; certaines de ces hypotheses paraissent même à la lumièce d'éludes récentes (1) (*) en contradiction avec des résultats expérimentaux.

(*) Les numéros entre parenthèses renvoient à la bibliographic. 
Une série de recherches se développèrent par ailleurs durant ces deux dernières décades autour du cas simple, imaginé par Taylon (2), de la turbulence homogène et isotrope. Karman el HowARTH (3) en précisèrent les bases théoriques et d'importants résultats furent obtenus, sans doute en partie grâce à la mise au point simultanée de la technique expérimentale de l'anémomètre à fil chaud qui permet de mesurer les valeurs des fluctuations de vitesse et des coefficients de corrélation.

Toutefois, ce type de turbulence, approximativement réalisé par l'écoulement derrière une grille, se rencontre rarement dans les problèmes abordés par l'Ingénieur; la plupart du temps, les ćcoulements qu'il étudie sont à gradient de vitesse et ni l'homogénéité ni l'isotropie ne viennent simplifier le champ de turbulence.

Malgré quelques tentatives intéressantes, aucune solution mathématique n'a été obtenue; ainsi peuvent s'expliquer les nombreuses recherches expérimentales effectués depuis quelques années; parmi les ćludes les plus importantes, nous citerons l'exploration de la couche limite le long d'une plaque plane dans le cas d'un gradient de pression nul par Townsend (4), KuEBANOFF et DreHL (5), et dans le cas d'un gradient de pression contraire par Schubauer, KleraNOFF (6) et CuAuser (7); l'étude de la couche limite, importante en vue des applications, est compliquée par la variation du nombre de Reynolds longitudinalement et surtout par le phénomène d'intermittence à la frontière entre le fluide libre et la couche limite; ce phénomène se présente aussi dans l'étude des jets, où il semble avoir ćté mis pour la première fois en évidence par Connsin (8), et dans les sillages, étudiés par
Townsend (9). L'écoulement en tuyau est au contraire affranchi de ces complications et LAUFER lui a consacré deux importantes études, d'abord en canal bi-dimensionnel (10), puis en tuyau circulaire (11).

L'étude de l'écoulement en canal divergent peut ètre rapprochée du travail de LAurer qu'elle étend au cas où le gradient de pression n'esl plus constant, et permet de s'échapper du caractère particulier que constitue pour les écoulements uniformes la distribution linéaire des efforts tangentiels (dans une même section, les accélérations différentes des filets fluides brisent cette loi); de plus, l'étude en canal bi-dimensionnel rapproche cet écoulement de celui d'une couche limite à gradient de pression contraire, tout en étant plus simple puisque le nombre de Reynolds reste constant d'une section à l'autre et que la fronlière libre intermittente est supprimée.

Ainsi avons-nous été amenés à reprendre les études de NikuRadze (12) sur les propriétés moyennes de l'écoulement divergent ef à les compléter, en faisant cette étude à l'air et en pouvant de ce fait utiliser l'anémomètre à fil chaud, par des mesures des fluctuations turbulentes des vitesses et des grandeurs statistiques associées à ces fluctuations.

Cette étude a été exéculée au Laboratoire de Mécanique des Fluides de l'Institut Polytechnique de Grenoble, sous le patronage du Service des Etudes el Recherches Hydrauliques d'Electricité de France. Nous sommes heureux de rendre ici hommage à M. Craya, professeur à la Faculté des Sciences, qui a proposé le sujet puis assuré la direction de cette rechercho.

\section{II. - LE TUNNEL ET . LES INSTRUMENTS DE MESURE}

\section{A) Le tunnel bi-dimensionnel.}

Une vue perspective du tunnel est donnée dans la figure 1; sa hauteur est de $80 \mathrm{~cm}$; sa largeur, de $3 \mathrm{~cm}$ à l'entrée, dépend à l'aval de la valeur de l'angle de divergence; les parois sont constituées par des plaques de duralumin parfaitement polies intérieurement.

L'air est aspiré à l'aval par l'intermédiaire d'un col sonique, fixant ainsi un débit rigoureusement constant et indépendant des fuctuations de la pompe d'aspiration; le débit à l'intérieur du tunnel est donc fonction de la section du col sonique, et un jeu de cols de sections différentes permet d'étudier des écoulements de débits différents. Le nombre de Reynolds $\mathrm{R}_{m}$ de l'écoulement, basé sur la vitesse moyenne $\mathrm{U}_{n}$ dans une section horizontale et sur la demi-largeur $h$ de cette section, n'est théoriquement fonction que

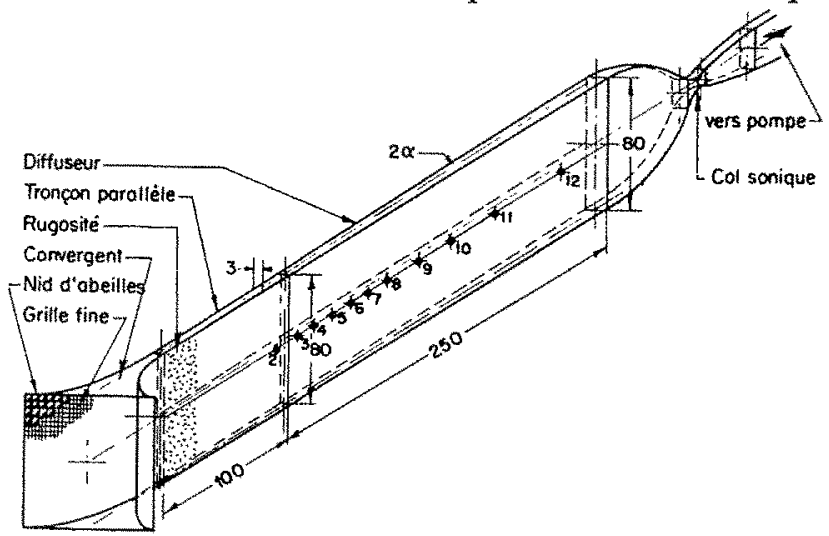

Fra. 1. - Schéma du tannel de mesure. 
du débit $Q$ aspiré; en effet, $l$ étant la hauteur du tunnel :

$$
\mathrm{R}_{m}=\frac{\mathrm{U}_{m} h}{\nu}=\frac{\mathrm{Q} h}{2 h l \nu}=\frac{\mathrm{Q}}{2 l \nu}
$$

il est en particulier indépendant de la largeur de la section et donc constant tout le long du tunnel.

Le tunnel comprend, de l'amont à l'aval :

1. Un convergent d'entrée dont le rapport de contraction latéral est égal à 30 , précédé d'un filtre du type nid d'abeille de maille carrée de $2 \mathrm{~cm}$ de côté et de $6 \mathrm{~cm}$ de profondeur, et d'une grille fine (maille de $0,8 \mathrm{~mm}$, fil de $0,3 \mathrm{~mm}$ de diamètre).

2. Un premier troncon à parois parallèles de $\mathbf{1} \mathbf{~ m}$ de longueur et de $3 \mathrm{~cm}$ de largeur, qui a pour but de permettre l'établissement d'un régime uniforme à l'entrée du divergent qui lui fait suite; la longueur de ce troncon n'étant toutefois pas suffisante pour obtenir à l'aval un régime uniforme nettement établi, nous avons collé, immédiatement à l'aval du convergent d'entrée et sur toute la hauteur du tunnel, une bande de papier rugueıx de $20 \mathrm{~cm}$ de longueur; cette rugosité a pour double effet, d'une part d'accélérer l'épaississement des couches limites développées à partir des parois latérales et d'obtenir ainsi un régime uniforme à l'entrée du divergent, d'autre part de fixer la transition entre couche limite laminaire et couche limite turbulente et d'éviter ainsi un déplacement incontrôlable de cette zone qui pourrait se répercuter sur l'écoulement à l'aval.

3. Un deuxième tronçon de $2,6 \mathrm{~m}$ de long qui constitue le divergent proprement dit; il est raccordé au précédent par des joints élastiques en tôle d'acier qui permettent de faire varier rapidement l'angle de divergence et assurent une continuité parfaite entre la surface des deux parois.

Dans le cas de l'angle de divergence de 6 degrés, pour éviter une trop srande largeur de section à l'aval du divergent où la bi-dimensionalité ne serait sans doute plus vérifiée, le deuxième tronçon est divisé en deux zones : la première, de $60 \mathrm{~cm}$ de longueur constitue le divergent; la seconde présente, comme le premier troncon, un écartement conslant; les diverses dispositions du tunnel sont schématisées sur la figure 2.

4. Un convergent conduisant au col sonique, luimême raccordé à l'exhausteur de la pompe par un divergent rond-carré.
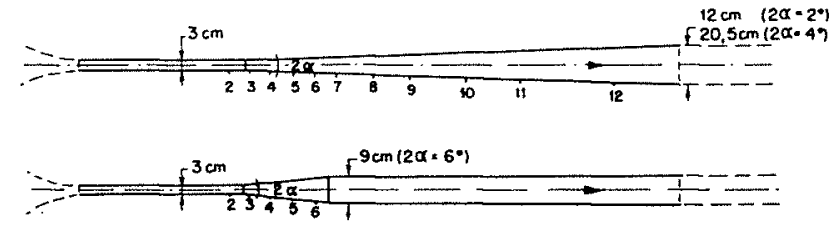

Fia. 2. - Plan du tunnel suivant les essais.

Les sections transversales de mesure sont situées dans le plan horizontal médian du tunnel. Dix sections (3 à 12) sont réparties le long du divergent; leurs distances $x$, à partir d'une origine définie par le point théorique d'intersection des prolongements des parois parallèles et des parois divergentes, sont rassemblées dans le lableau ci-dessous :

\begin{tabular}{c|c|c|c|c|c|c} 
Section & 2 & 3 & + & 5 & 6 & 7 \\
\hline $\mathrm{X}_{\mathrm{cm}}$ & -11 & 3,5 & 15,4 & 30,3 & 44,4 & 58,6
\end{tabular}

\begin{tabular}{l|c|c|c|c|c} 
Section & 8 & 9 & 10 & 11 & 12 \\
\hline $\mathrm{X}_{\mathrm{cm}}$ & 76,7 & 94,8 & 124,8 & 154,8 & 204,8
\end{tabular}

En outre, dans le plan vertical des sections 3 et 6 , plusieurs autres sections de mesure ont été ménagées afin d'étudier l'écoulement de part et d'autre du plan horizontal de symétrie du tunne] et de contrôler la bi-dimensionalité de l'écoulement.

\section{B) Instruments de mesure.}

Dans chaque section nous avons tout d'abord mesuré les répartitions des vitesses moyennes à l'aide d'ume sonde de pression totale, la pression statique étant prise à la paroi; nous avons ensuite mesuré à l'anćmomc̀lre à fil chaud, outre les vitesses moyennes, les répartitions des fluctuations longitudinales des vitesses el diverses grandeurs caractérisant la turbulence (dérivées et fonction spectrale des fluctuations longitudinales).

\section{Mesure des vitesses MOYennes aU PITOT.}

La pression statique est mesuréc à la paroi, par l'intermédiaire de deux prises de $0,5 \mathrm{~mm}$ de diamètre percées au droit de chaque section de mesure; la pression dynamique est donnée par une sonde hypodermique (de diametre intérieur égal à $0,6 \mathrm{~mm}$ ) fixée à un système de traverséc essentiellement conslitué par une vis micrométrique permettant d'apprécier facilement un déplacement transversal de la sonde à 1/100 de $\mathrm{mm}$ près; les manometres utilisés, soit du type Casella, soit du type Fortier, assurent une précision de $1 / 20^{\circ}$ de $\mathrm{mm}$ sur la valeur de la hauteur. manométrique.

L'erreur relative sur les valeurs des vitesses ainsi déterminées (due à l'erreur sur la hauteur 
manométrique) est de $\pm 1 / 100$ pour une vitesse de $6 \mathrm{~m} / \mathrm{s}$ et diminue lorsque la vitesse augmente (elle est de $\pm 1 / 1000$ pour une vitesse de $18,4 \mathrm{~m} / \mathrm{s}$ ); la gamme des vitesses que nous avons mesurées s'étend entre 6 el $45 \mathrm{~m} / \mathrm{s}$. La précision sur les distributions des vitesses relatives est done supérieure à $1 / 100$ et augmente rapidement pour les points situés dans le centre de la section; pour les valeurs absolnes de vitesses, il faut, en plus de l'erreur sur la hauteur manométrique, tenir compte de l'erreur sur les valeurs de la densité du liquide manométrique et de l'air; dans tous les cas, l'erreur relative totale n'a pas dépassé $1 / 100$.

Deux corrections ont été apportées à ces mesures; d'une part une correction due au gradient de vitesse au droit de la prise de pression dynamique qui a pour effet de déplacer « le centre effectif $》$ de la sonde vers les vitesses les plus élevées; nous arons, pour calculer ce déplacement, utilisé la formule de Young et Mass (13):

$$
\delta / D=0,131+0,082 \mathrm{D}_{1} / \mathrm{D}
$$

dans laquelle $\mathrm{D}$ et $\mathrm{D}_{2}$ sont les diamètres extérieur et intérieur de la sonde; dans notre cas, suivant la section de mesure, cette correction sur les valeurs de $y / h$ varie de 0,012 à 0,0035 .

La seconde correction lient compte de l'effet de turbulence sur la pression totale; on montre facilement que si $U_{n}$ est la vitesse moyenne mesurée, $\pi^{2}$ la valeur quadratique moyenne de la fluctuation longitudinale de cette vitesse, la vavaleur exacte $U$ de la vitesse est en première approximation :

$$
\mathrm{U}=\mathrm{U}_{m}\left(1-\frac{\overline{a^{2}}}{\mathrm{U}_{m^{2}}}\right.
$$

Utilisant les valeurs de $\bar{u}^{2}$ mesurées avec l'anémomètre à fil chaud, nous avons trouvé que cette correction est maximum et égale à $3 \%$ pour les points situés près de la paroi et diminue rapidement vers le centre de la section; nous en avons tenu compte dans nos résultats.

\section{MFsuRES A L'ANÉMOMETRE A FIL CHAUD,}

L'appareil utilisé est un anémomètre à fil chaud à température constante; nous voulons ici remercier $M$. le professeur $H$. Rovsr, directeur de l'Institut de Recherches Hydrauliques d'lowa, qui nous a obligeamment communiqué les schémas de l'appareil concy par un de ses collaborateurs, M. le professeur P. Hubbard; M. Elbeno, ingénieur radio-électricien au Laboratoire de Grenoble, a construit et mis au point lappareil.

Nous rappellerons brièvement le principe de l'anémomètre à fil chaud. Un fil fin, chauffé par un courant électrique, est placé dans le courant d'air à ćtudier (son axe perpendiculaire à la vitesse moyenne de l'écoulement). L'aix dissipe la chaleur du fil par convection forcée et l'expérience montre que la quantité de chaleur $\mathrm{H}$ ainsi dissipée est proportionnelle, d'une part à la différence entre la température du fil 0 et la température de l'air ambiant $\theta_{a}$, d'autre part à la racine carrée de la vitesse de lair $\mathrm{V}$ :

$$
H=(A+B \sqrt{V})\left(0-\cdots 0_{0}\right)
$$

le coefficient A tenant compte des pertes de chaleur du fil par radiation et convection libre.

La température du fil étant liée à sa résistance électrique $R$ par la relation :

$$
\mathrm{R}=\mathrm{R}_{t i}\left[1+\alpha\left(0 \cdots 0_{a}\right)\right]
$$

$\mathrm{R}$ et $\mathrm{R}_{a l}$ étant les valeur's de la résistance du fil

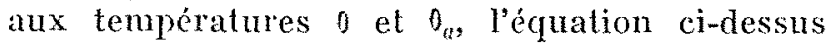
s'ecrit :

$$
H=(A+B \sqrt{ }) \frac{R-R_{n}}{\% R_{a}}
$$

La chaleur est fournie au fil par un courant d'intensité I; en supposant que le fil n'emmagasine pas de chaleur, l'égalité entre les quantités de chaleur recue et dissipée par le fil s'écrit :

$$
\mathrm{RI}^{2}=(\mathrm{A}+\mathrm{B} \sqrt{\mathrm{V}})\left(\frac{\mathrm{R}-\mathrm{R}_{\eta}}{\alpha \mathrm{R}_{i}}\right) \text { (formule de King) }
$$

dans laquelle $R$, I et $V$ sont des variables.

Dans l'appareil à température constante, la résistance du fil (done sa température) est maintenue constante quelle que soil la vitesse $\mathrm{V}$ et la mesure de l'intensité I du courant traversant le fil donne, grâce à un étalomnage préalable, la valeur de la vitesse $V$.

Si la vitesse $V^{\top}$ fluctue (régime turbulent), l'intensité I du courant fluctuc également; le fil étant maintenu pratiquement à température constante, son inertie thermique est négligcable jusqu'à des fréquences de fluctuation élevées et les fluctuations d'intensité correspondent en amplitude et en phase aux fluctuations de vitesse (contrairement à ce qui se passe avec un fil chaud du type a intensité constante ou l'inertie thermique doit être compensce par un élage électroni(que spécial).

Schématiquement, le monlage à température constante comprend essentiellement (fig. 3) :

- Un pont de Wheatstone dans l'une des branches duquel est place le fil chaud;

-.. Une alimentation représentée schc̉matiquement par la lampe $\mathrm{E}_{2}$, alimentant le pont par la diagonale AD; à l'ćquilibre du pont, la différence de potentiel entre $B$ et $C$ est nulle; s'il y a déséquilibre, une tension $e$ apparaît entre $B$ et $C$; 


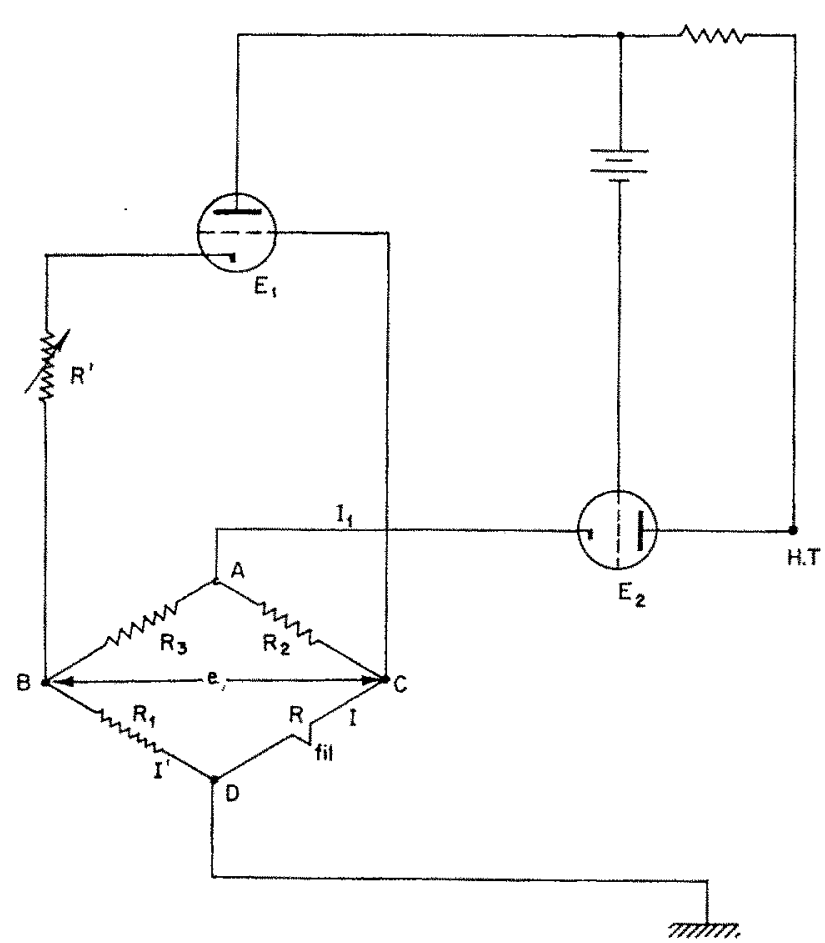

Frg. 3. - Schéma de principe de l'anémomètre à fil chaud à température constante.

- Un amplificateur représenté schématiquement par la lampe $E_{1}$ dans lequel est injectée la tension $e$ et qui, agissant sur la grille de la lampe de puissance, modifie le courant d'alimentation $I_{1}$ du pont, de telle facon que $e$ redevienne nulle;

- Enfin, un étage (non représenté sur la figure 3) qui, amplifiant les variations de $I_{1}$, en mesure la valeur moyenne dans un systime thermocouple-milivoltmetre.

L'appareil que nous avons construit permet de mesurer, sans distorsion, des fuctuations jusqu'à une fréquence de $10000 \mathrm{~Hz}$. Le fil utilisé est en platine, d'une longueur de $0,5 \mathrm{~mm}$ et de 4 microns de diamètre; il est soudé entre deux aiguilles de laiton, elles-mêmes montées dans une sonde en matiere isolante; cette sonde est déplacée dans la transversale de mesure par le mécanisme de traversée déjà décrit.
L'appareil ainsi équipé d'un fil simple permet de mesurex, d'une part la vitesse moyenne $\mathbf{U}$ de l'air au droit du fil, d'autre part la valeur quadratique moyenne $u^{\prime}$ de la fuctuation longitudinale de vitesse $u\left(u^{\prime}=\sqrt{\overline{\left.u^{2}\right)}}\right.$. Grâce à un double étase dérivaleur, nous pouvons mesurer de plus les valeurs quadratiques moyennes de la dérivée premièe $\partial u / \partial t$ et seconde $\partial^{2} u / \partial t^{2}$ de la fluctuation $u$. Un autre étage, construit spécialement à Grenoble, permet de mesurer la valeur quadratique moyenne des puissances troisiome et quatrième des dérivées :

$$
\overline{\left(\frac{\partial u}{\partial t}\right)^{3}} \text { et }\left(\frac{\partial u}{\partial t}\right)^{4} \text { ou } \overline{\left(\frac{\partial^{2} u}{\partial t^{2}}\right)^{3}} \text { et }\left(\frac{\partial^{2} t}{\partial t^{2}}\right)^{4}
$$

Enfin, nous verrons plus en détail, dans le paragraphe consacré à la structure fine de la furbulence, qu'en envoyant le signal dectrique donné par le fil chaud dans un analyseur d'onde, on peut déterminer la fonction spectrale de la fluctuation $u$.

Nous n'entrerons pas ici dans le détail d'une correction dite de «longneur de fil », qui tient compte de l'influence de la longueur finie du fil par rapport a la longueur d'onde des lluctuations (14); nous l'avons négligée dans la mesure de $u^{\prime}$, son ordre de grandeur étant, pour nos essais, inférieur à $1 \%$; par contre, cette correction n'est pas négligeable pour la mesure des valeurs de la fonction spectrale correspondant aux fréquences élevées (elle varie, suivant les sections, entre $4 \%$ et $10 \%$ pour la fréquence de $10000 \mathrm{~Hz}$ ), de même que pour la valeur de l'échelle de turbulence que l'on déduit du spectre (elle varie entre $3 \%$ et $10 \%$, suivant les sections de mesure). Dans les résultats présentés ci-après, les valeurs de la fonction spectrale ne sont pas corrigées; par contre, les valeurs de $\lambda$ sont corrigées.

Il est difficile de chifrer exactement la précision des mesures effectuces avec l'anémomètre a fil chaud (15); néanmoins, d'après la dispersion des points de mesure, on pent estimer que lerreur sur les valeurs de $\|^{\prime}$ est inférienre $\pm 5 \%$.

\section{III. - RÉSULTATS ET DISCUSSION}

\section{A) Généralités.}

Nous avons étudié successivement l'écoulement dans des divergents d'angle total 2 a égal à 2,4 et 6 degrés; le nombre de Reynolds $\mathrm{R}_{\mathrm{m}}=\mathrm{U}_{m} \mathrm{~h} / \mathrm{v}$ est, pour les trois essais, égal à $32000\left(^{*}\right)$.

(*) Dans s cas du divergent d'angle total 2 degrés, nous avons de plus étudie l'ecoulement pour un nombre
Nous ayons vérifić, grâce atux sections prérues à cet efret, que l'écoulement conserve des

de Reynolds égal at 52.000 ; nous n'examinetons pas ici cet essat dont les resultats onl de prescntes au $6^{*}$ Comgres de l'A.I.R.H. dans une communication relative it l'ecoulement turbulent dans un divergent bi-dimensionnel d'angle total égal a 2 degres, pour les deux nombres de Reynolds de 32.000 et 52.000 . 
propriétés identiques dans les transversales de mesure situées de part et d'autre de la transversale du plan horizontal nédian; autrement dit, l'écoulement est bien bi-dimensionnel dans la partie centrale du tunnel. Nous devons toutefois signaler que le nombre de Reynolds défini ci-dessus, qui devrait être constant d'une section à l'autre, augmente en fait vers l'aval; cette circonstance paraìt inévitablement liée à la hauteur finie du tunnel et correspond à l'influence du frottement sur les parois inférieures et supérieures du tunnel qui entraîne une accélération de la tranche centrale de l'écoulement; les valeurs de $\mathrm{R}_{m}$ indiquées ci-dessus sont les valeurs moyennes pour le régime d'équilibre.

I'écoulement a été étudié jusqu'aux sections 12 pour l'angle de 2 degrés, 9 pour l'angle de 4 degrés et 6 pour l'angle de 6 degrés.

Dans un premier paragraphe sont jrésentés les résultats concernant l'écoulement moyen
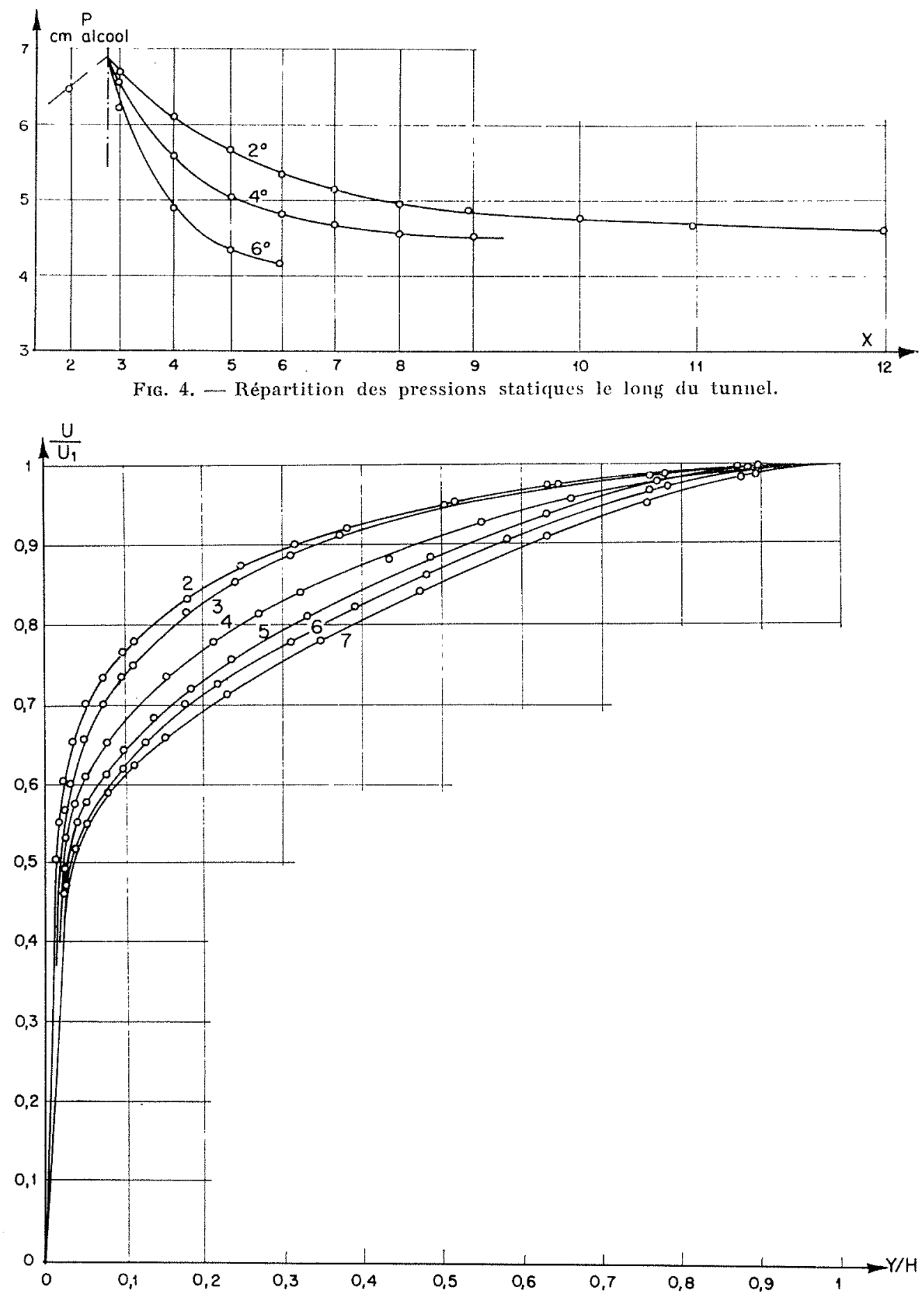

FiG. 5. - Distribution des vitesses moyennes; angle $2 \alpha=2^{\circ}$; sections 2 it 7 . 
(pression statique le long du tunnel, vitesses moyennes dans les différentes transversales de mesure) et diverses grandeurs qui s'en déduisent (vitesse de frottement à la paroi, loi logarithmique de vitesse, répartitions des tensions de Reynolds longitudinales, perte d'énergie du mouvement moyen par unité de longueur) ainsi que les répartitions, dans les différentes transversales de mesure, des moyennes quadratiques des fluctuations longitudinales de vitesse.

Dans une seconde partie, les caractéristiques de la structure fine de la turbulence (fonctions spectrales des fluctuations longitudinales, dissipation de l'énergie turbulente, échelles de turbulence, corrélations triples en un point) sont plus particulièrement étudiées.

\section{B) Ecoulement moyen et fluctuations longitu- dinales de vitesse.}

\section{FCOUlement MOYEN.}

La figure 4 indique les répartitions des pressions statiques le long du tunnel pour les dif- férents angles de divergence, la différence entre la pression à la section considérée et la pression à l'entrée du tunnel (exprimée en cm d'alcool, de densité égale à 0,815 ) étant portée en ordonnée, et les distances de chaque section comptées à partir de la première section de mesure en abscisse.

Sur les figures 5 et 6 sont tracées les répartitions des vitesses moyennes dans les onze transversales de mesure, pour l'essai correspondant à l'angle de divergence de 2 degrés; en ordonnée est porté le rapport $U / U_{1}$ de la vitesse moyenne $U$ en un point situé à la distance $y$ de la paroi à la vilesse moyenne $U_{1}$ au centro de la section, en abscisse le rapport $y / h$ ( $h$ étant la demi-largeur de la section considérée); nous n'avons représenté que le demi-profll des vitesses, l'écoulement étant symétrique par rapport au plan vertical médian du tunnel.

On remarque que les profils des vitesses des sections les plus à l'aval $(10,11$ et 12) se groupent autour d'une courbe unique; l'éconlement atteint donc, après une zone de transition (sec-

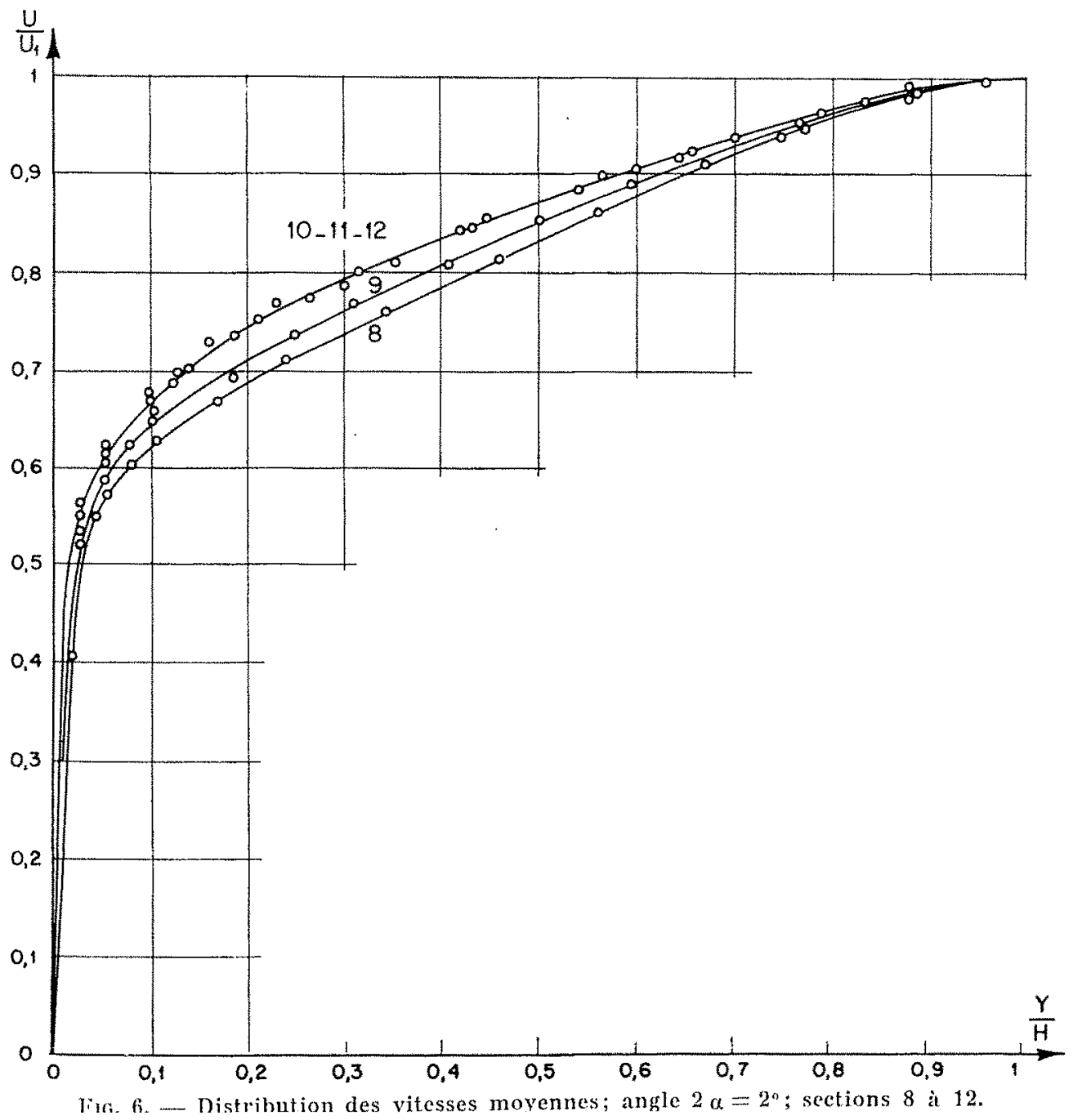




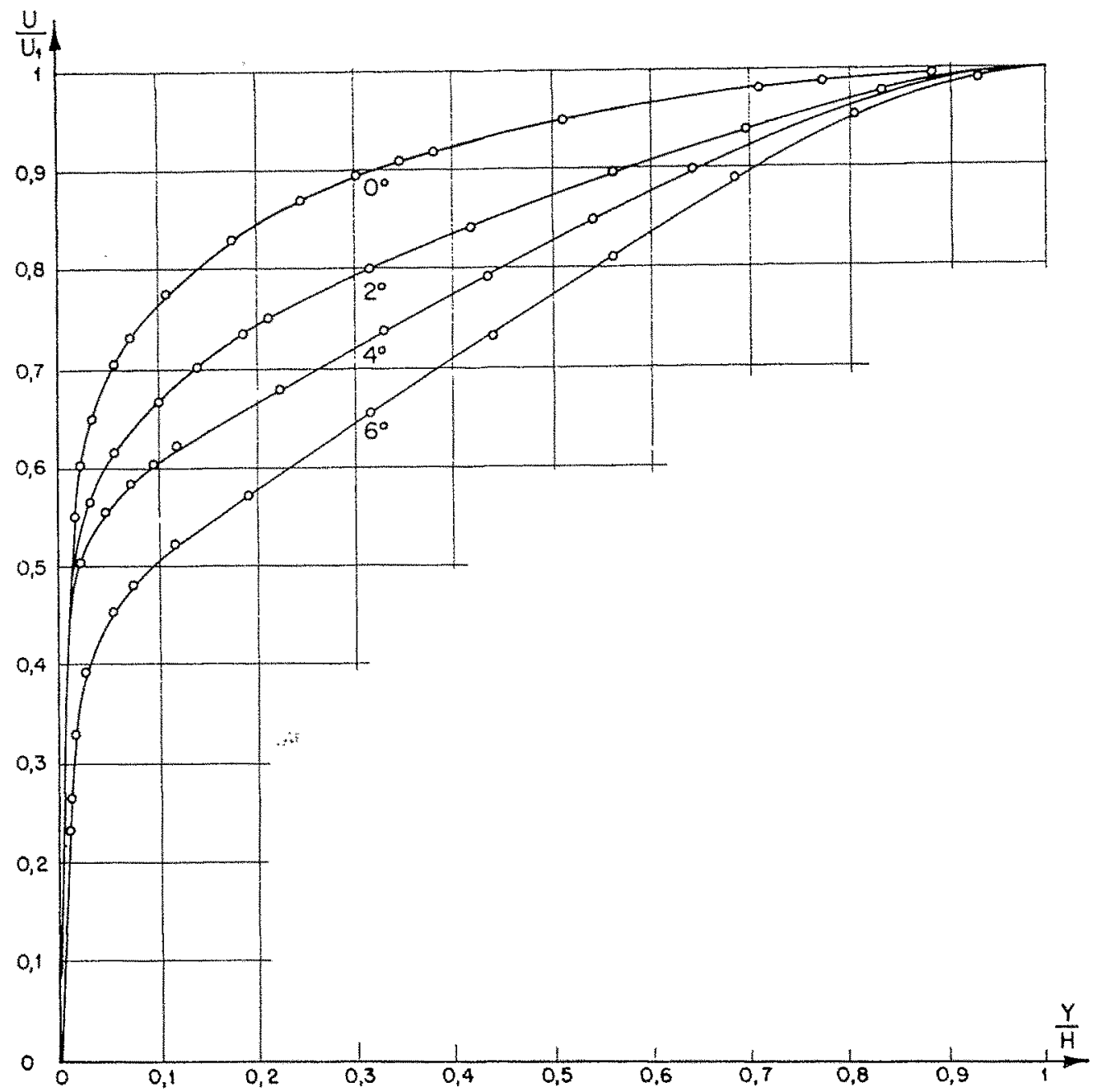

Frg. 7. - Distribution des vitesses moyennes pour les diffẻrents régimes d'équilibre.

tions 3 à 9), un régime d'équilibre caractérisé par une affinité des distributions des vitesses moyennes dans les différentes transversales de mesure; nous reviendrons plus loin sur cette propriété, qui est également vérifiée pour les angles de divergence de 4 et 6 degrés.

Les répartitions des vitesses dans chaque transversale de mesure ont été explorées pour tous les angles de divergence, mais faute de pouvoir présenter tous les résultats dans le cadre limité de cette note, nous n'avons tracé sur la figure 7 que les profils d'équilibre des écoulements pour les différents angles de divergence, ainsi que la répartition des vitesses du régime uniforme entre parois parallèles (section 2); ces résultats sont en bon accord avec ceux obtenus par NukuRadze dans un divergent parcouru par de l'eau.

La figure 8 représente, pour un des essais effectués, les courbes de répartition des vitesses au voisinage de la paroi $(0<y / h<0,1)$; grâce à l'anémomètre à fil chaud, on a pu mesurer les vitesses jusqu'à une distance de $1 / 10^{\circ}$ de $\mathrm{mm}$ de la paroi et atteindre ainsi le film laminaire pour lequel les points représentatifs des vitesses sont sensiblement alignés sur une demi-droite passant par l'origine.

On peut calculer directement la vilesse de frottement $\mathrm{U}_{*}$ dans chaque section de mesure à partir de la valeur $p$ de la pente de la droite représentative des vitesses dans le film laminaire; en effet, cette vitesse est définie à partir de la force de frottement $\tau_{0}$ à la paroi par unité de surface par la relation : $\boldsymbol{U}_{*}=\sqrt{\tau_{0} / 6}$; or, en régime laminaire :

$$
\tau=u \frac{d u}{d y}=\mu \times p \times \frac{\mathrm{U}_{1}}{h}
$$

I’où :

$$
\mathrm{U}_{*}=\sqrt{\frac{v U_{1}}{h} \times p}
$$

nous avons porté les valeurs de $\mathrm{U}_{*}$ ainsi déterminées pour les différents essais sur la figure 9.

Nous avons tracé sur la figure 10 les répartitions des vitesses $U / U_{*}$ en fonction du loga- 


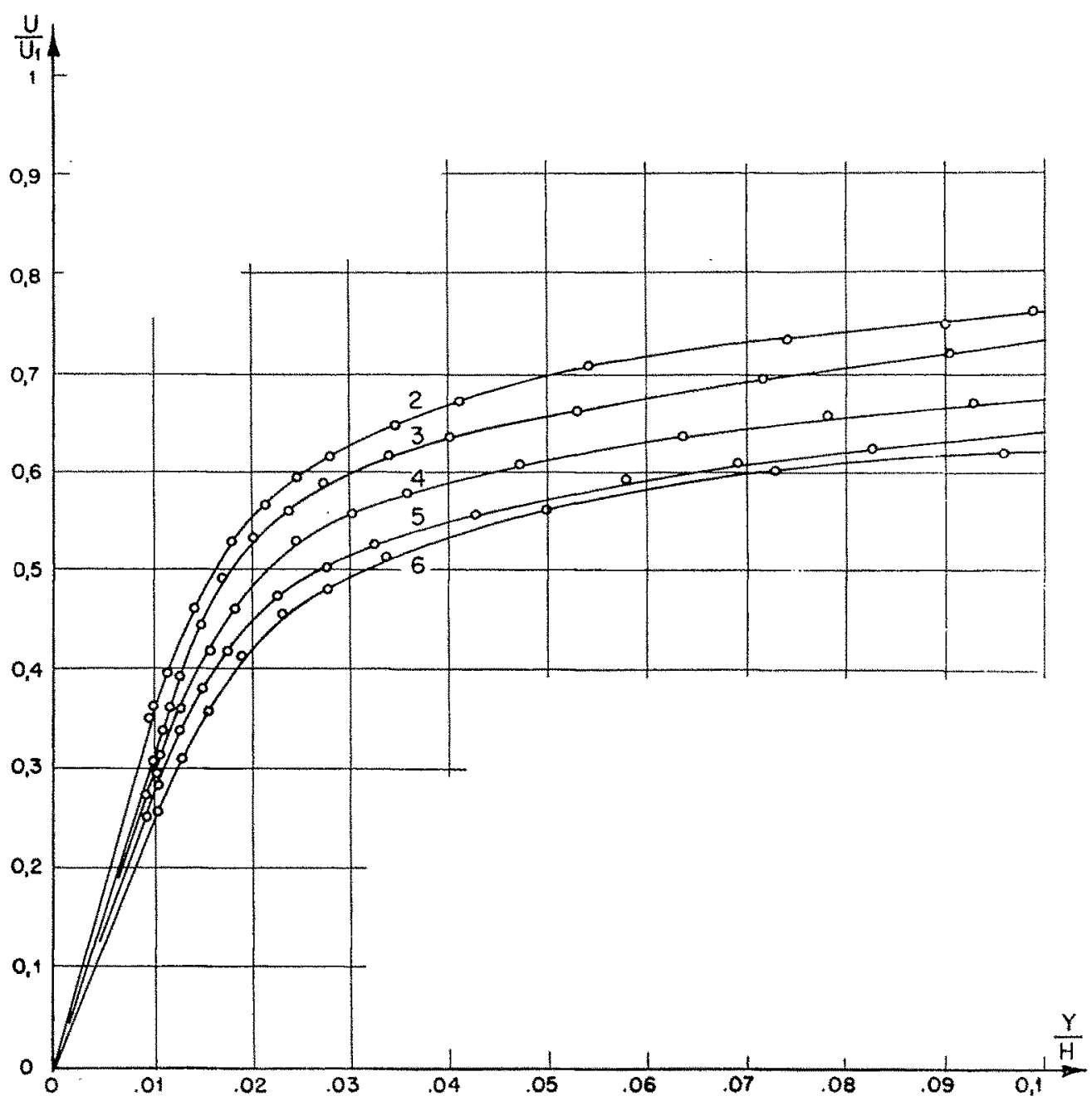

FIG. 8. - Distribution des vitesses moyennes près de la paroi; angle $2 \alpha=2^{\circ}$; sections 2 a 6 .

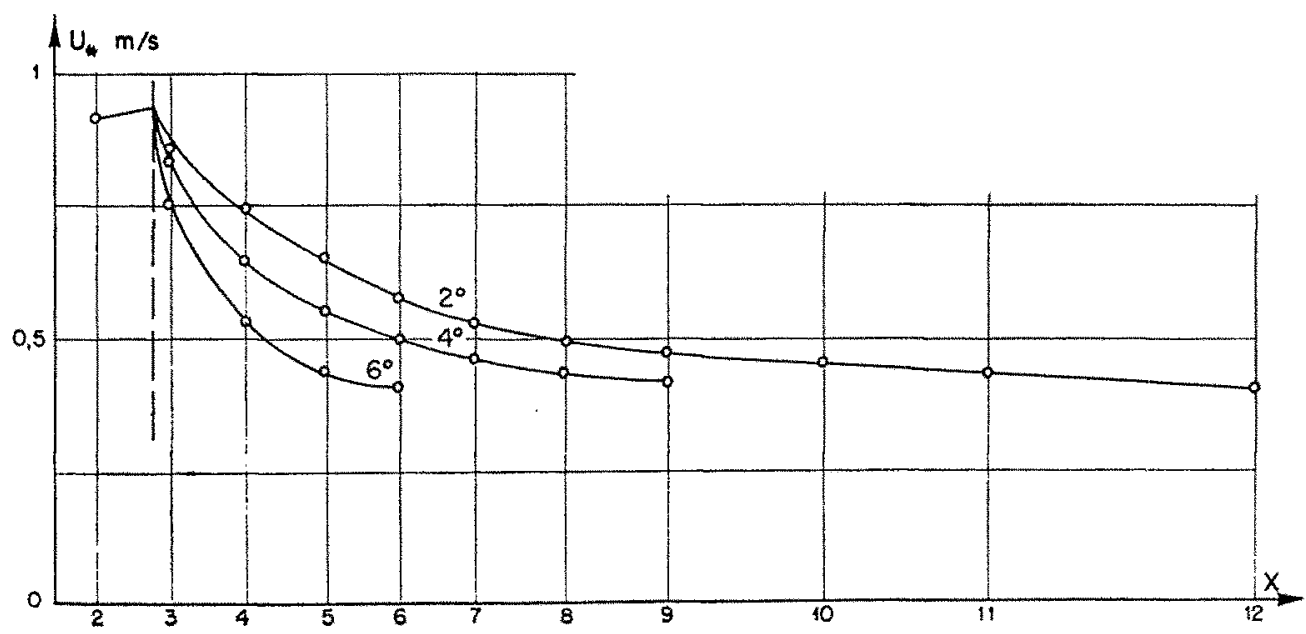

Fig. 9. - Répartition des vitesses de frottement le long du tunnel. 


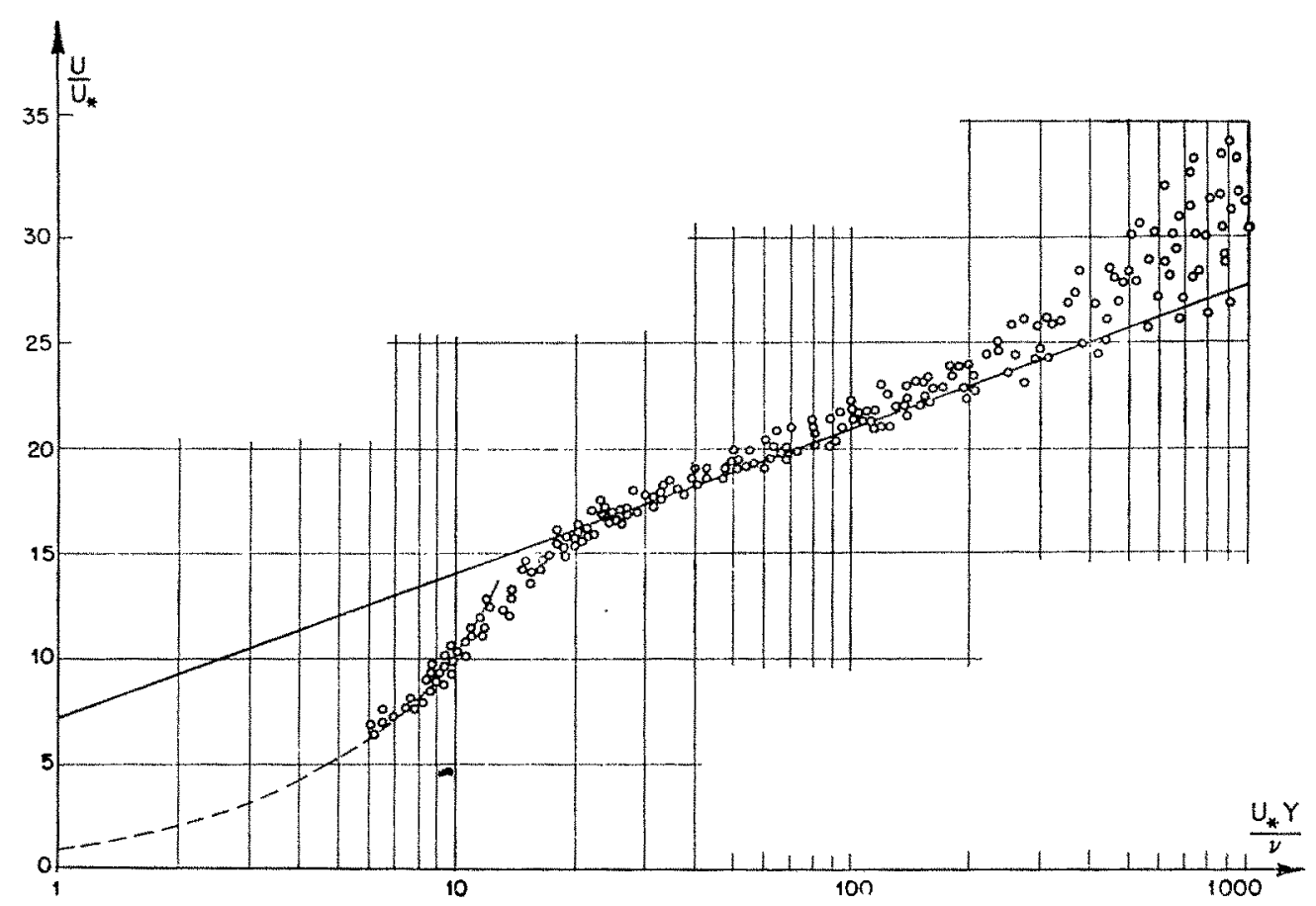

Fia. 10. - Distribution des vitesses; angle $2 \alpha=2^{\circ}$; sections 2 i 12 .

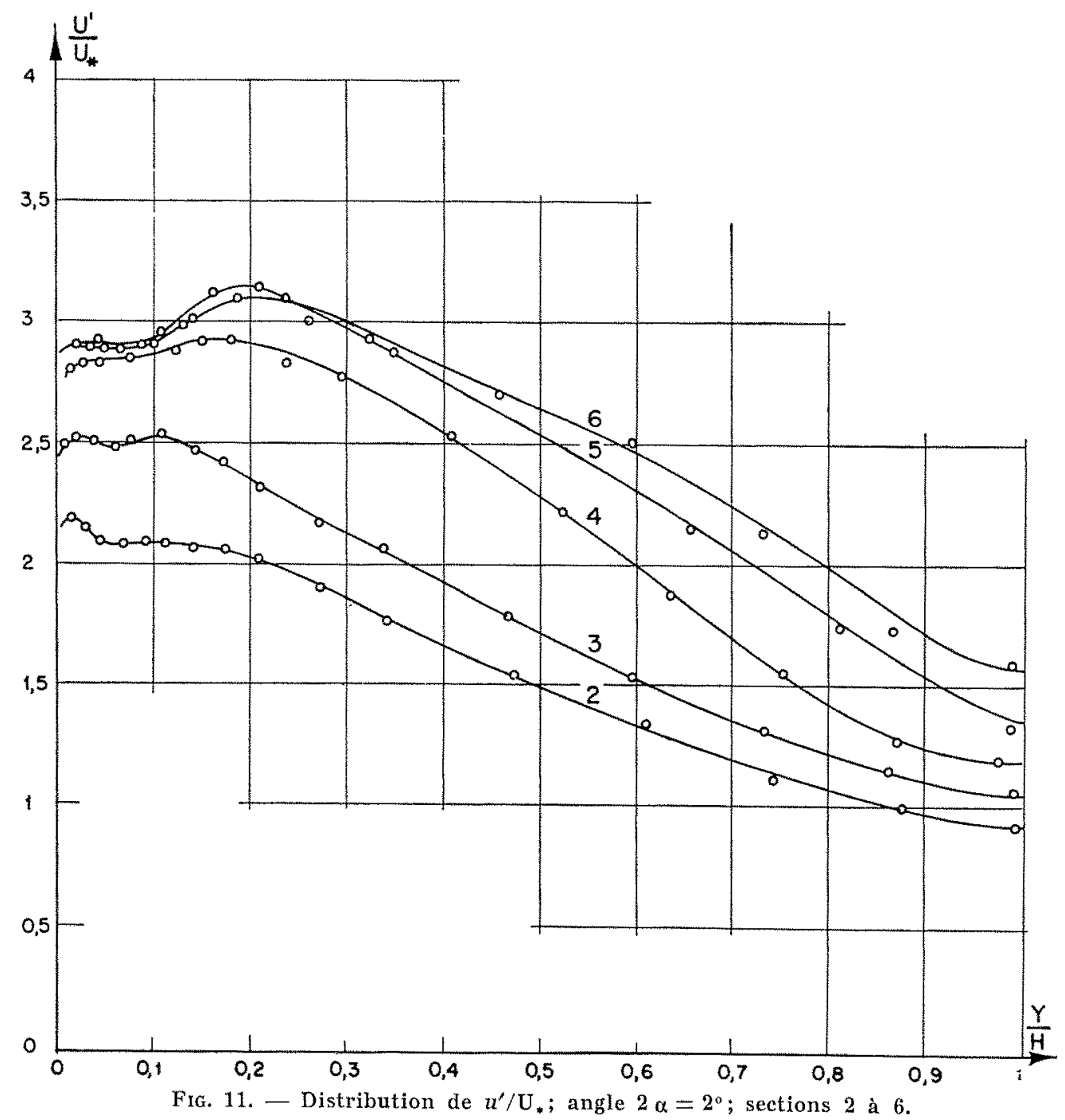


rithme de $U_{*} y / \nu$ pour les différentes sections, dans le cas de l'angle total de divergence de 2 degrés. Si la loi de Karman :

$$
\frac{\mathrm{U}}{\mathrm{U}_{*}}=\mathrm{A} \log \frac{\mathrm{U}_{*} y}{v}+\mathrm{B}
$$

est bien vérifiée pour l'écoulement uniforme entre parois parallèles (section 2), elle ne l'est plus pour le centre de la section, dans le cas de l'écoulement dans un divergent (section 3 à 12); en effet, une des hypothèses de base de la théorie de Karman est que la distribution des tensions tangentielles soit linéaire; or cette loi n'est plus exacte dans le divergent, en raison de l'existence des forces d'accélération. Il est toutefois intéressant de noter que près de la paroi :

$$
\left(20<\frac{\mathrm{U}_{*} y}{v}<200\right)
$$

la loi logarithmique est vérifiée, quel que soit le type d'écoulement; très près de la paroi, dans le film laminaire, la distribution des vitesses suit la loi $\mathrm{U} / \mathrm{U}_{*}=\mathrm{U}_{*} y / \nu$.

Les valeurs des coefficients $A$ et $B$ sont respectivement égales à 6,9 et 7,25 environ; ces valeurs sont en accord avec celles trouvées par LAUFER pour un écoulement uniforme dans un canal à parois parallèles, mais différent des valeurs obtenues par NikuRAdze pour des écoulements en tuyaux circulaires.

Des résultats analogues ont été obtenus pour les autres angles de divergence étudiés.

\section{Flucituations longitudinales de vitesse.}

Nous avons vu que l'anémomètre à fil chaud permet de mesurer la moyenne quadratique $\boldsymbol{u}^{\prime}$ de la fluctuation longitudinale de vitesse $u$; le niveau local de turbulence en un point étant une des composantes $d u$ tenseur de Reynolds en ce point, il est logique de rapporter la valeur de $u^{\prime}$ en un point d'une section à la vitesse de frottement relative à cette section. Nous avons ainsi tracé sur les figures 11 et 12 les courbes de

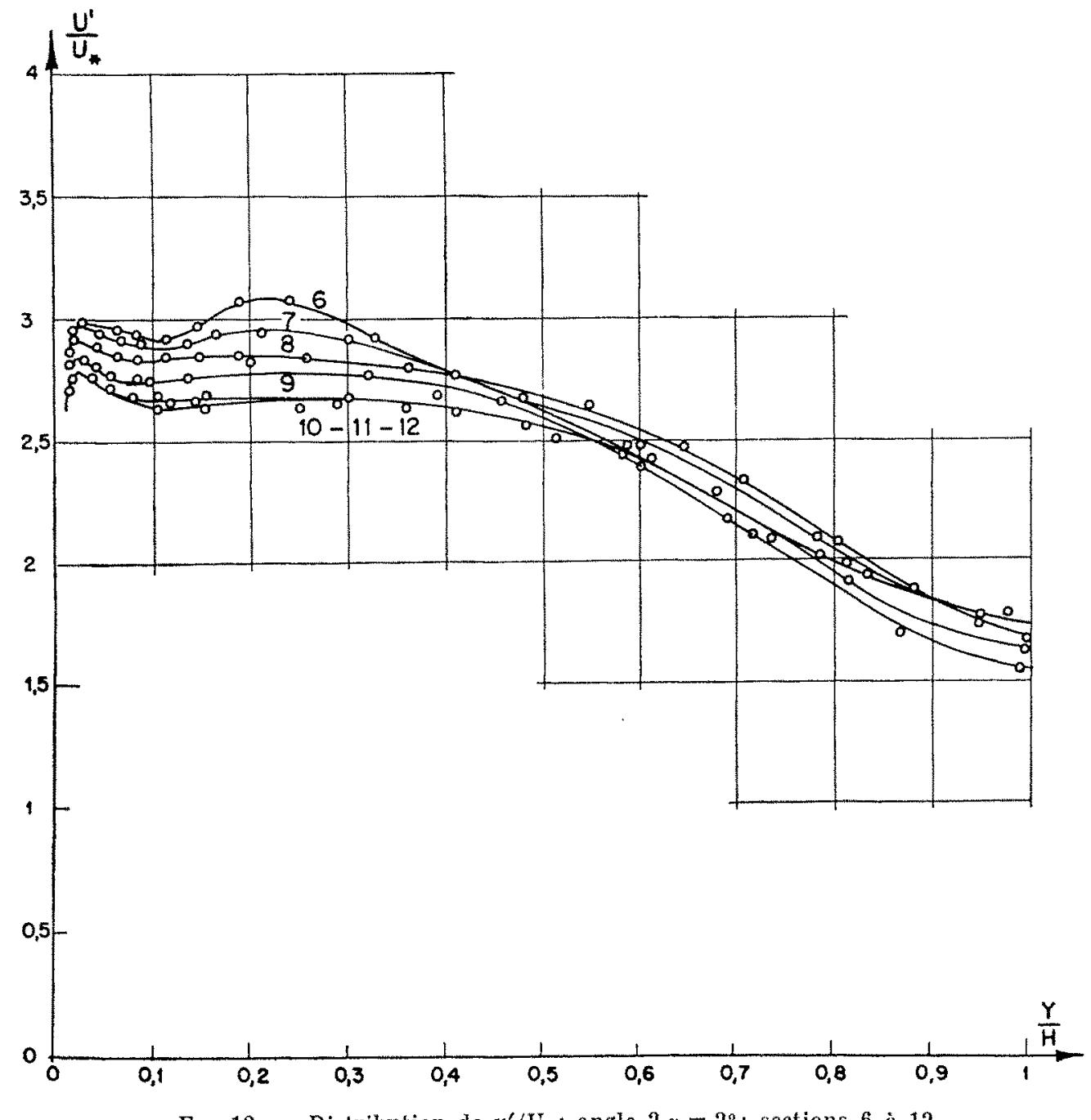

FIG. 12. - Distribution de $u^{*} / \mathrm{U}_{*}$; angle $2 \alpha=2^{\circ}$; sections 6 à 12 . 


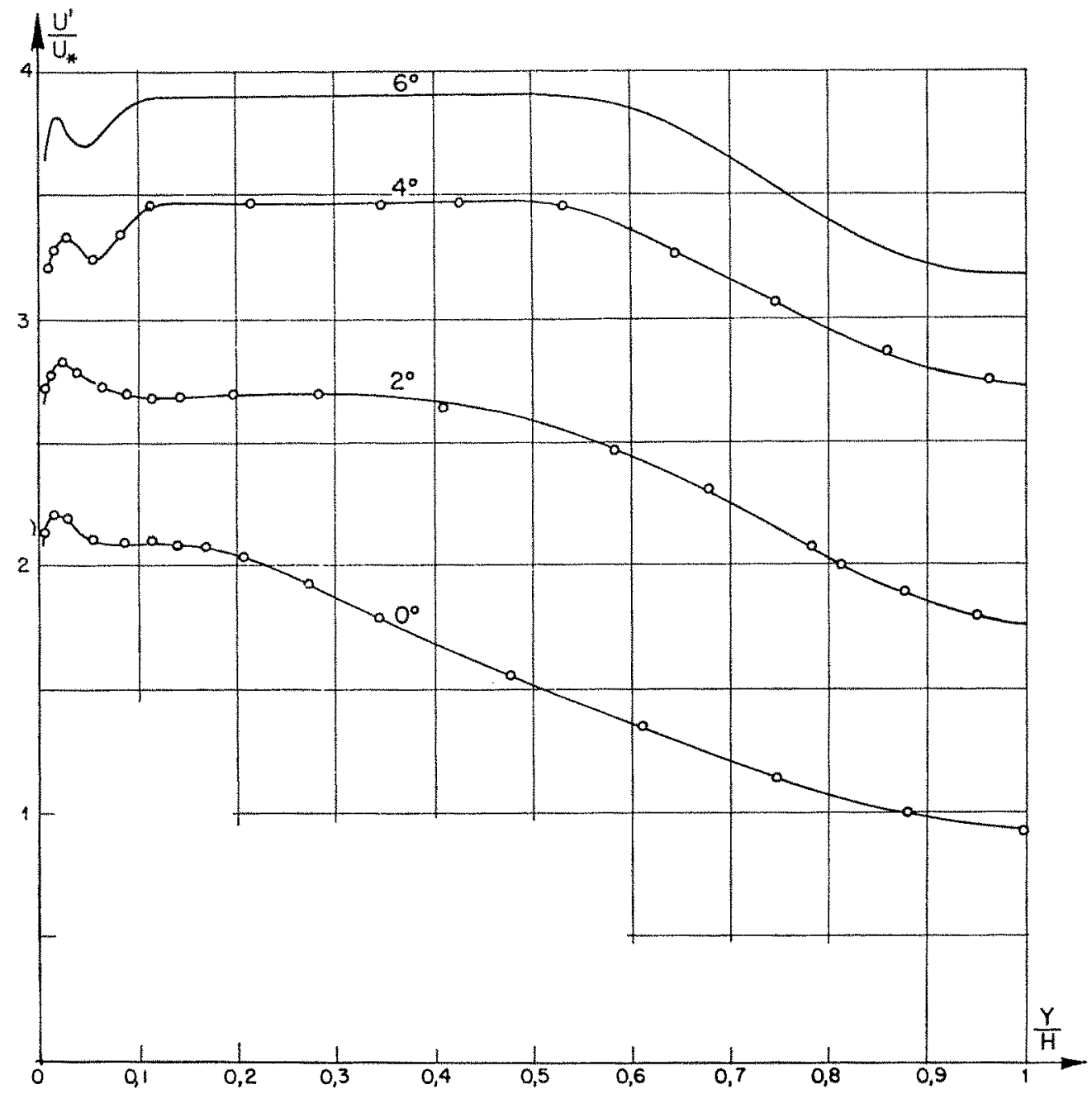

Frg. 13. - Distribution de $u^{\prime} / U_{*}$ pour les différents régimes d'équilibre.

répartition de $u^{\prime} / \mathrm{U}_{*}$ en fonction de $y / h$ pour les différentes transversales de mesure, dans le cas de l'angle de divergence de $2^{\circ}$.

Il ressort de cette figure qu'à partir de la section 10 les valeurs de $u^{\prime} / \mathrm{U}_{*}$ se groupent (comme c'était le cas pour les vitesses moyennes) autour d'une courbe unique, définissant ainsi, comme nous le verrons plus loin, une répartition caractéristique d'un régime d'équilibre.

Nous avons tracé sur la figure 13 les répartitions de $u^{\prime} / \mathrm{U}_{*}$ correspondant aux régimes d'équilibre obtenus pour les différents angles de divergence étudiés, ainsi que la répartition à la section 2; on notera l'augmentation progressive de l'énergie turbulente en fonction de la valeur de l'angle de divergence, cet accroissement étant lié à la décélération de plus en plus grande de l'écoulement.

\section{Etude thÉorique du RÉGIME d'ÉQuiLibRe.}

Les résultats expérimentaux ont montré l'exis- tence dans la partie aval du divergent d'un régime caractérisé par une similitude des courbes de répartition des vitesses moyennes et des fluctuations longitudinales de vitesses rapportées à la vitesse de frottement. Nous allons voir qu'en supposant l'écoulement radial on peut prévoir théoriquement l'existence d'un tel régime d'équilibre.

En effet, avec cette hypothèse, les équations de Reynolds, en un point de coordonnées polaires $r, \varphi$, s'écrivent (16) :

$$
\begin{gathered}
\frac{\partial p / \rho}{\partial r}=-\mathrm{U} \frac{\partial \mathrm{U}}{\partial r}-\left(\frac{\partial \bar{u}^{2}}{\partial r}+\frac{1}{r} \frac{\partial \bar{u} \bar{v}}{\partial \varphi}+\frac{\bar{u}^{2}-\bar{v}^{2}}{r}\right) \\
+v \frac{1}{r^{2}} \frac{\partial^{2} \mathrm{U}}{\partial \varphi^{2}} \\
\frac{\alpha}{\varphi}
\end{gathered}
$$

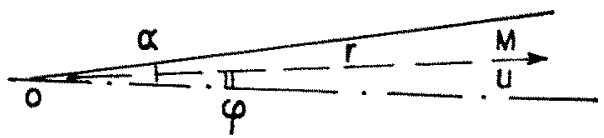




$$
\begin{array}{r}
\frac{1}{r} \frac{\partial p / \rho}{\partial \varphi}=-\left(\frac{\partial \pi v}{\partial r}+\frac{1}{r} \frac{\partial v^{2}}{\partial \varphi}+\frac{2 \pi v}{r}\right) \\
+v \frac{2}{r^{2}} \frac{\partial \mathrm{U}}{\partial \varphi}
\end{array}
$$

et l'équation de continuité : $\partial / \partial r(r \mathrm{U})=0$, $U$ étant la vitesse moyenne en $M$ suivant $O M$,

$p$ la pression moyenne en M,

$u$ et $v$ les fluctuations de vitesse en $M$, suivant OM et la perpendiculaire à OM.

De l'équation de continuité résulte immédiatement que :

$$
\begin{aligned}
& r \mathrm{U}=g(\varphi) \\
& r \mathrm{U}_{1}=g(\alpha)
\end{aligned}
$$

( $U_{1}$ étant la vilesse moyenne sur l'axe).

D'oil : $\mathrm{U}=\mathrm{U}_{1} f(p)$.

La courbe de répartition des vitesses :

$$
\frac{\mathrm{U}}{\mathrm{U}_{1}}=f(\varphi)
$$

est donc indépendante de $r$.

En remplaçant U par $\mathrm{U}_{1} f(\%)$ dans les équations de Reynolds, on obtient:

$$
\begin{aligned}
& \frac{r}{\mathrm{U}_{1}{ }^{2}} \frac{\partial p / 0}{\partial r}=f^{2}-\frac{r}{\mathrm{U}_{1}^{2}} \\
& \left(\frac{\partial \pi^{2}}{\partial r}+\frac{1}{r^{r}} \frac{\partial \pi v}{\partial \varphi}+\frac{\overline{u^{2}}-\overline{v^{2}}}{r}\right)+\frac{v}{\mathrm{U}_{1} r} f^{\prime \prime} \\
& \frac{r}{U_{1}^{2}} \frac{\partial p / Q}{\partial \varphi}=-\frac{r}{U_{1}^{2}} \\
& \left(\frac{\partial \bar{u}}{\partial r}+\frac{1}{r} \frac{\partial \bar{v}^{2}}{\partial \varphi}+\frac{2 \overline{u v}}{r}\right)+\frac{2 v}{U_{1} r} f^{\prime}
\end{aligned}
$$

ot on remarque qu'il suffl que:

$$
\frac{\overline{u^{2}}}{\mathrm{U}_{1}^{2}}, \frac{\overline{v^{2}}}{\mathrm{U}_{1}^{2}}, \frac{\overline{w^{2}}}{\mathrm{U}_{1}^{2}}
$$

no dépendent que de pour que les seconds membres de ces équations soient indépendants de $r$.

Les équations de Reynolds étant encore valables dans le film laminaire, on a :

$$
\mathrm{U}_{*}^{2}=\frac{\tau_{0}}{\varphi}=\frac{v}{r}\left(\frac{\partial \mathrm{U}}{\partial \varphi}\right)_{\varphi=0}=\frac{v \mathrm{U}_{i}}{r}, f_{\varphi=0}^{*}(\varphi)
$$

d'où :

$$
\frac{\mathrm{U}_{*}^{2}}{\mathrm{U}_{1}^{2}}=\frac{y}{\mathrm{U}_{1} r} \cdot f_{\dot{\gamma}=0}^{\prime}(\varphi)=\mathrm{C}^{\mathrm{to}}
$$

Une condition suffisante pour qu'un régime d'équilibre puisse exister, est donc que les quantités :

$$
\frac{\overline{a^{2}}}{\mathrm{U}_{*}^{2}}, \frac{\overline{v^{2}}}{\mathrm{U}_{*}^{2}}, \frac{\overline{u v}}{\mathrm{U}_{*}^{2}}
$$

soient indépendantes de $r$.

L'expérience a montré qu'il en était bien ainsi pour les valeurs de $\widetilde{u}^{2} / U_{*}^{2}$, tout au moins à l'aval d'une zone de transition dans laquelle l'hypothèse de radialité n'est sans doute pas vérifiée.

\section{RÉPARTITION DES TENSIONS TANGENTIELLES ET PERTE D'ÉNERGE DU MOUVEMENT MOYEN.}

Nous avons vu que l'intensité de turbulence dans un divergent est plus grande que dans un canal plan, et ceci d'autant plus que l'angle de divergence est grand; cet accroissement de turbulence tient à cc que les différents filets fluides n'ont pas, dans un divergent, les mêmes taux d'accélération, ce qui entraîne comme nous allons le voir des tensions de Reynolds plus grandes et une perte d'énergie du mouvement moyen plus élevée.

Plaçons-nous dans le cas du régime d'équilibre; l'équation de Reynolds en axes rectangulaires ( $O x$ étant l'axe du divergent) s'écrit :

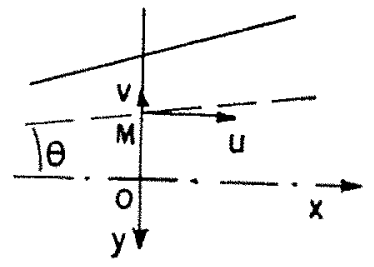

$$
\frac{1}{\rho} \cdot \frac{\partial p}{\partial x}=-\left(\mathrm{U} \frac{\partial U}{\partial x}+\mathrm{V} \frac{\partial U}{\partial y}\right)+\frac{1}{\rho} \frac{\partial \tau}{\partial y}
$$

dans laquelle $U$ et $V$ sont les projections de la vitesse moyenne en $M$ sur $O x$ et $O y$, et :

$$
\tau=-\rho \overline{u U}+t \cdot \frac{\partial U}{\partial y} .
$$

la tension tangentielle ( $n$ el $y$ ctant les mroiections de la fluctualion de vitesse sur $O x$ et $O y$ ).

Calculons le terme d'accélération U:

$$
\mathrm{U} \frac{\partial \mathbf{U}}{\partial x}+\mathrm{V} \frac{\partial \mathbf{U}}{\partial y}
$$

en remarquant successivement que l'écoulement étant radial $\mathrm{U} / \mathrm{U}_{1}=g(\eta)(\eta=y / h)$, que, d'après la figure ci-dessus :

$$
V=-0 \lg 0 \quad\left(\operatorname{tg} \theta=\frac{h-y}{\gamma}\right)
$$


et qu'enfin, d'après l'équation de continuité :

$$
\frac{\partial \mathrm{U}}{\partial \boldsymbol{x}}+\frac{\partial \mathrm{V}}{\partial y}=0 ; \text { d'où : }
$$

$\mathrm{U} \frac{\partial \mathrm{U}}{\partial x}+\mathrm{V} \frac{\partial \mathrm{U}}{\partial y}=-\mathrm{U} \frac{\partial \mathrm{V}}{\partial y}+\mathrm{V} \frac{\partial \mathrm{U}}{\partial y}=$

$-U \frac{\partial-(U \operatorname{tg} \theta)}{\partial y}-U \operatorname{tg} 0 \frac{\partial U}{\partial y}=-\frac{U^{2}}{h} \operatorname{tg} \alpha$

L'équation du mouvement s'écrit alors:

$$
\frac{1}{f} \frac{\partial \tau}{\partial y}=\frac{1}{p} \frac{\partial p}{\partial x}-\frac{\mathrm{U}^{2}}{h} \operatorname{tg} \alpha
$$

soit :

$$
\frac{\partial \tau / \rho U_{1}^{2}}{\partial \eta}=-\operatorname{tg} \alpha\left(g^{2}-\frac{h}{\rho U_{1}^{2} \operatorname{tg} \alpha} \cdot \frac{\partial p}{\partial x}\right)
$$

En posant :

$$
M=\frac{h}{\rho U_{1}^{2} \operatorname{tg} \alpha} \cdot \frac{\partial p}{\partial x}
$$

et en remarquant que d'après les hypothèses sur la similitude longitudinale $M$ ne dépend que de $\alpha$, on peut intégrer l'équation ci-dessus.

$$
\frac{\tau}{\rho U_{1}^{2}}=\operatorname{tg} \alpha \int_{\eta}^{1} g^{2} d \eta-M \operatorname{tg} \alpha(1-\eta)
$$

Or, pour $n=0$

$$
\tau=\tau_{0}=\rho U_{1}^{2} \operatorname{tg} \alpha(b-M) \quad\left(b=\int_{0}^{1} g^{2} d x_{1}\right)
$$

d'où':

$$
\mathrm{M}=b-\frac{\tau_{0}}{i \mathrm{U}^{2} \operatorname{tg} \alpha}=b-\frac{1}{\operatorname{tg} \alpha} \frac{\mathrm{U}_{*}^{2}}{\mathrm{U}_{1}{ }^{2}}
$$

et :

$$
\frac{\tau}{\rho U_{1}^{2}}=\frac{U_{*}^{2}}{U_{1}{ }^{2}}(1-\eta)+\operatorname{tg} \alpha \int_{\eta}^{1}\left(g^{2}-b\right) d \eta
$$

soit encore :

$$
\frac{\tau}{\rho U_{1}^{2}}=\frac{U_{*}^{2}}{U_{1}^{2}}(1-\eta)+\operatorname{tg} \alpha \int_{0}^{\eta}\left(b-g^{2}\right) d \eta
$$

en effet

$$
\int_{\eta}^{1}=\int_{0}^{1}-\int_{0}^{\eta}=0-\int_{0}^{\eta}
$$

d'où finalement :

$$
\frac{\tau}{\tau_{0}}=1-\eta+\frac{\mathrm{U}_{1}^{2} \operatorname{tg} \alpha}{\mathrm{U}_{*}{ }^{2}} \int_{0}^{\eta}\left(b-g^{2}\right) d \eta
$$

Le supplément de tension tangentielle par rapport à un canal plan pour lequel $\tau / \tau_{0}=1-\eta_{\text {, }}$ est égal à :

$$
\mathrm{U}_{1}^{2} \operatorname{tg} \alpha . \int_{0}^{\eta}\left(b-g^{2}\right) d \eta
$$

Les répartitions de $\tau / \tau_{0}$ en fonction de $y / h$ pour les différents divergents étudiẻs, calculées' à partir de la formule ci-dessus et des courbes de répartition des vitesses moyennes, sont groupées sur la figure 14.

L'énergie du monvement moyen, perdue dans un tronçon du tumnel de longueur unité, est égale à la somme de l'énergie directement dissipée en chaleur par ce mouvement moyen :

$$
\left(\int_{0}^{2 k} v\left(\frac{\partial U}{\partial y}\right)^{2} d y\right)
$$

et de la production d'énergie turbulente :

$$
\left(\int_{0}^{2 \hbar}-\bar{u} \bar{v} \frac{\partial \mathrm{U}}{\partial y} d y\right)
$$

soit :

$$
\mathrm{E}=\int_{0}^{\rho n}\left(-\pi \overline{\Pi \nu}+\nu \frac{\partial \mathrm{U}}{\partial y}\right) \frac{\partial \mathrm{U}}{\partial y} d y
$$

Or :

$$
\tau=-\rho \bar{u} \bar{v}+\mu \frac{\partial U}{\partial y},
$$

d'où :

$$
\frac{\mathbf{E}}{\mathbf{2}}=\int_{0}^{h} \frac{\tau}{\rho} \frac{\partial U}{\partial y} d y
$$

Nous calculerons cette dernière intégrale par parties, en remarquant que $\tau=0$ pour $y=h$ et $\mathrm{U}=0$ pour $y=0$ :

$$
\begin{aligned}
\int_{0}^{h} & \frac{\tau}{\rho} \frac{\partial \mathrm{U}}{\partial y} d y=\left(\frac{\tau}{\rho} \mathrm{U}\right)_{0}^{h} \\
& -\int_{0}^{h} \mathrm{U} \frac{\partial \tau / \rho}{\partial y} d y=-\int_{0}^{h} \mathrm{U} \frac{\partial \tau / \rho}{\partial y} d y
\end{aligned}
$$

or :

$$
\begin{aligned}
\mathrm{U} \frac{\partial \tau / \rho}{\partial y}=\frac{\mathrm{U}_{1}^{3}}{h} \cdot \frac{\mathrm{U}}{\mathrm{U}_{1}} \cdot \frac{\partial \tau / \rho \mathrm{U}_{1}{ }^{2}}{\partial \eta}= \\
\quad-\frac{\mathrm{U}_{1}{ }^{3}}{h} \cdot \operatorname{tg} \alpha \cdot g\left(g^{2}-\mathrm{M}\right)
\end{aligned}
$$

d'où :

$\int_{0}^{h} \mathrm{U} \frac{\partial \tau / p}{\partial y} d y=-\frac{\mathrm{U}_{1}^{3}}{h} \operatorname{tg} \propto \int_{0}^{h} g\left(g^{2}-\mathrm{M}\right) d y$ $=-\mathrm{U}_{1}{ }^{3} \lg \alpha \int_{0}^{\perp} g\left(g^{2}-\mathrm{M}\right) d \gamma_{1}$;

en posant :

$$
\int_{0}^{1} \frac{\mathrm{U}}{\mathrm{U}_{1}} d \eta=a, \quad \int_{0}^{1}\left(\frac{\mathrm{U}}{\mathrm{U}_{1}}\right)^{3} d \eta=c
$$

et en remplaçant $M$ par sa valeur :

$$
\left(\mathrm{M}=b-\frac{\mathrm{U}_{*} / \mathrm{U}_{1}{ }^{2}}{\operatorname{tg} \alpha}\right)
$$




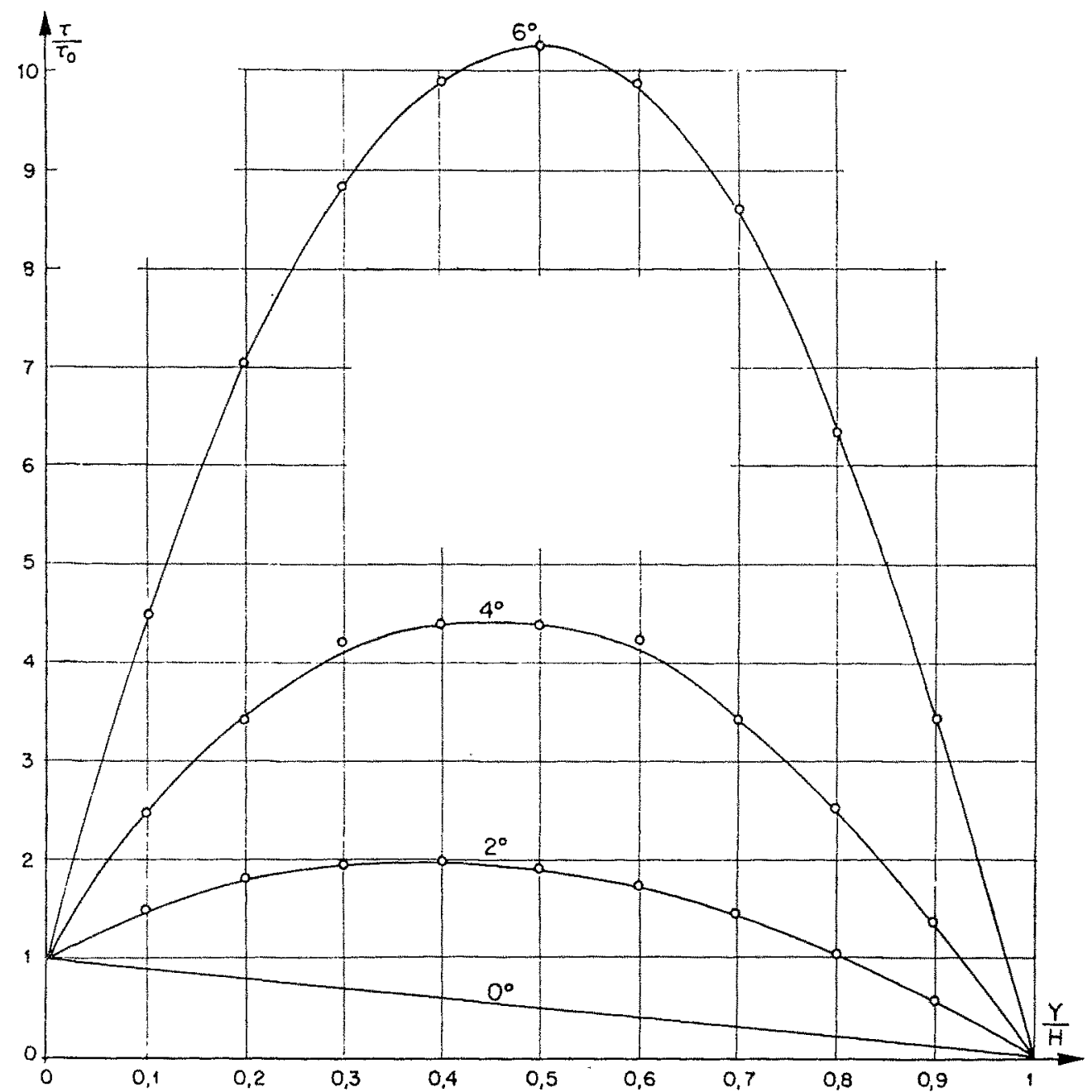

Fıg. 14. - Distribution des tensions tangentielles pour les différents régimes d'équilibre.

il vient après simplification:

$$
-\int_{0}^{h} \mathrm{U} \frac{\partial \tau / \rho}{\partial y} d y=a \mathrm{U}_{1} \mathrm{U}_{*}^{*}+\mathrm{U}_{1}^{3} \operatorname{tg} \alpha(c-a b)
$$

Dans un canal à parois parallèles, l'énergie dissipée $\mathrm{F}_{0}$ est égale à $2 \alpha \mathrm{U}_{1} \mathrm{U}_{*}:$; pour un canal divergent et pour un même nombre de Reynolds de l'écoulement, le supplément d'énergie dissipée, égal à $2(c-a b) \mathbf{U}_{1}{ }^{3} \operatorname{tg} \alpha$, peut être caractérisé par le rapport :

$$
k=\frac{(c-a b) \mathrm{U}_{1}: t^{\prime} x}{a \mathrm{U}_{1} \mathrm{U}_{*}^{3}}
$$

d'après nos essais, les valeurs de $k$ sont respectivement égales à $0,37,1,03$ et 3,05 pour les angles de divergence de 2,4 et 6 degrés.

Ce calcul met en évidence l'importance des pertes d'énergie dans les écoulements divergents, cette énergie se retrouvant principalement sous forme d'énergie turbulente.

\section{C) Structure fine de la turbulence.}

1. Définition de la fonction SPECTRALE, DES ÉCHELLES ET MICROÉCHELLES DE TURBULENCE.

Jusqu'ici, nous avons caractérisé la turbulence en un point par la moyenne quadratique de la fluctuation longitudinale de vilesse $u^{\prime}=\sqrt{\overline{u^{2}}}$; $u^{\prime 2}$ représente l'énergie turbulente en ce point dans le sens de l'écoulement.

Pour analyser plus en détail la fluctuation $u(t)$, on peut avoir recours à l'analyse harmonique; on peut par exemple considérer la fluctuation $u(t)$ comme étant une somme de fluctuations primaires de fréquences différentes $n$ et déterminer la valeur quadratique moyenne de ces fluctuations primaires en fonction de leur fréquence.

On effectue généralement cette mesure en envoyant le signal électrique sortant de l'amplificateur basse fréquence du fil chaud dans un analyseur d'onde qui ne laisse passer que les 
fluctuations de courant dont la fréquence est comprise dans une bande $n-n+d n, n$ pouvant varier dans des limites étendues (nous avons utilisé dans cette étude un analyseur "General Radio, type $736 \mathrm{~A}$ » dont la bande passante a une largeur de $5,36 \mathrm{~Hz}$ et la gamme d'exploration est comprise entre 20 et $16.000 \mathrm{~Hz}$ ); le signal à la sortie de l'analyseur est envoyé dans un cireuit comprenant en série un thermocouple et un millivoltmètre et on détermine ainsi sa valeur quadratique moyenne. La valeur de la fluctuation primaire par unité d'intervalle de fréquence est obtenue en divisant la valeur mesurée par la largeur de la bande passante.

On peut donc déterminer par cette méthode une fonction $f(n)$ représentant la fraction d'énergie de la fluctuation longitudinale de vitesse associée à la fréquence $n$, et l'on a :

$$
\int_{0}^{\infty} f(n) d n=u^{\prime 2}
$$

En général, on remplace la fréquence $n$ par le nombre d'onde $k_{1}$, défini par $k_{1}=\left(2 \pi_{t} / \mathrm{U}\right)$ (U étant la vitesse moyenne au point considéré), et on exprime la distribution spectrale de l'énergie de fluctuation longitudinale par la fonction $\mathrm{F}\left(k_{1}\right)$, normalisée par la relation :

$$
\int_{0}^{\infty} \mathrm{F}\left(k_{1}\right) d k_{1}=1
$$

On sait que, dans un écoulement turbulent, l'énergie dissipée en chaleur se décompose en deux parties: d'une part l'énergie dissipée parles forces de viscosité dues au mouvement moyen [nous avons vu ci-dessus que dans une section cette énergie est donnée par l'intégrale $\left.\int_{0}^{2 h} \mu(\partial U / \partial y)^{2} d y\right]$, d'autre part, l'énergie dissipée par les fluctuations turbulentes, dont l'expression est donnée par la fonction de Rayleigh :

$$
\begin{aligned}
& \mathrm{W}=2 \mu\left[\left(\frac{\partial u}{\partial x}\right)^{2}+\left(\frac{\partial \bar{v}}{\partial y}\right)^{2}+\left(\frac{\partial w}{\partial z}\right)^{2}\right. \\
& +\frac{1}{2}\left(\frac{\partial v}{\partial z}+\frac{\partial w}{\partial y}\right)^{2}+\frac{1}{2}\left(\frac{\partial w}{\partial x}+\frac{\partial \bar{u}}{\partial z}\right)^{2} \\
& +\overline{\frac{1}{2}\left(\frac{\partial u}{\partial y}+\frac{\partial v}{\partial x}\right)^{2}}
\end{aligned}
$$

qui se réduit, dans le cas où la turbulence est isotrope, $\dot{a} \mathrm{~W}=15 u \overline{(\partial u / d x)^{2}}$. La fonction spectrale $\mathrm{F}\left(k_{1}\right)$ permet de connaître, avec quelques hypothèses, la quantité d'énergie dissipée en chaleur par la turbulence. En effet, sans reprendre le raisonnement exposé pour la première fois par TAYlor (17), nous rappellerons seulement qu'une quantité telle que $(\partial u / \partial x)^{2}$ peut être liće à la valeur limite du coefficient de corrélation entre les fluctuations de vitesse de deux points infiniment voisins, et que ce coefficient de corrélation est lui-même lié à la fonction spectrale; en bref, on a la relation :

$$
\frac{1}{\bar{u}^{2}} \overline{\left(\frac{\partial u}{\partial x}\right)^{2}}=\int_{0}^{\infty} k_{1}^{2} \mathbf{F}\left(k_{1}\right) d k_{1}
$$

Si l'intégrale du moment de deuxième ordre de la fonction spectrale ne donne pas dans notre cas la valeur de l'énergie dissipée par la turbulence [puisque, la turbulence n'étant pas isotrope, la fonction de Rayleigh ne se réduit pas au seul terme $\left.\overline{(\partial u / \partial x)^{2}}\right]$, l'allure de la courbe $k_{1}{ }^{2} \mathrm{~F}\left(k_{1}\right)$ en fonction de $k_{1}$ indique néanmoins la gamme de fréquence qui contribue le plus à cette dissipation.

On définit une longueur caractéristique de turbulence (appelée généralement microéchelle) par la relation $\lambda=u^{\prime} /\left[\overline{(\partial u / \partial x)^{2}}\right]^{1 / 2}$. Cette échelle est ainsi reliée directement à la dissipation d'énergie turbulente; on peut la déterminer soit à partir de la fonction spectrale (par intégration du moment du deuxic̀me ordre), soit par mesure directe de $\bar{\pi}^{2}$ et $\overline{(\partial u / \partial x)^{2}}$; ayant étudié systématiquement dans nos essais les fonctions spectrales, nous avons déterminé $\lambda$ par la premic̀re méthode; quelques mesures par la seconde méthode ont confirmé les résultats obtenus.

On définit une autre échelle de turbulence (macro-échelle), qui correspond qualitativement à la longueur d'onde des plus' gros tourbillons, par la relation :

$$
\mathrm{L}_{x}=\int_{0}^{\infty} \frac{\overline{u_{0} u_{x}}}{{\overline{u_{0}}}^{2}} d x
$$

( $u_{0}$ et $u_{x}$ étant les fluctuations longitudinales de vitesse en deux points d'abscisse 0 et $x$ ), ou par la relation équivalente :

$$
\mathrm{L}_{x}=\frac{\pi}{2} \mathrm{~F}(0)
$$

Nous avons estimé $L_{x}$ par la seconde formule en extrapolant la fonction $\mathrm{F}\left(\boldsymbol{k}_{1}\right)$ jusqu'à $k_{1}=0$.

\section{Résultats des mesurfes.}

Nous avons mesuré pour chaque angle de divergence la fonction spectrale $F\left(k_{1}\right)$ en cind points de chacune des sections de mesure; la distance relative de ces points à la paroi, $y / h$, est en général égale à 0,$02 ; 0,25 ; 0,50 ; 0,75$ et 1 . Nous avons tracé sur la figure 15 les résultats obtenus pour une section d'équilibre de l'écoulement dans le divergent d'angle total égal à 2 degrés. On remarque qu'une grande part de l'énergie est associée aux fluctuations de faibles nombres d'onde; pour le point central de cette 
section, par exemple, $85 \%$ de l'énergie est fournie par des fluctuations de fréquence inférieure à $500 \mathrm{~Hz}$ et $98 \%$ par des lluctuations de fréquence inférieure à $700 \mathrm{~Hz}$.

Par contre, les courbes des moments du deuxième ordre des fonctions spectrales (fig. 16) indiquent que ce sont les fluctuations de fréquences élevées qui dissipent le plus d'énergie; c'est ainsi que pour le point déjà envisagé cidessus, $18 \%$ seulement de l'énergie est dissipée par des fluctuations de fréquence inférieure à $500 \mathrm{~Hz}$.

Nous ne donnerons pas, faute de place, les courbes correspondant aux autres sections et aux autres angles de divergence; nous indiquerons seulement ci-après les valeurs des échelles de turbulence que l'on en déduit.

Sur le graphique 17 sont tracées les répartitions des microéchelles $\lambda$ rapportées à la demilargeur $h$ de la section pour les différentes transversales du divergent d'angle total égal à 2 degrés; on remarque que ces répartitions sont également affines dans la zone d'équilibre définie au chapitre précédent. Sur la figure 18, nous avons tracé les répartitions $\lambda / h$ correspondant aux régimes d'équilibre des' divers angles de divergence étudiés : pour un point situé à une même distance relative de la paroi, le rapport $\lambda / h$ décroît lorsque l'angle de divergence augmente, traduisant ainsi l'augmentation de la dissipation d'énergie. Les valeurs des échelles $L_{x}$ de turbulence (fig. 19) sont de l'ordre de gran-

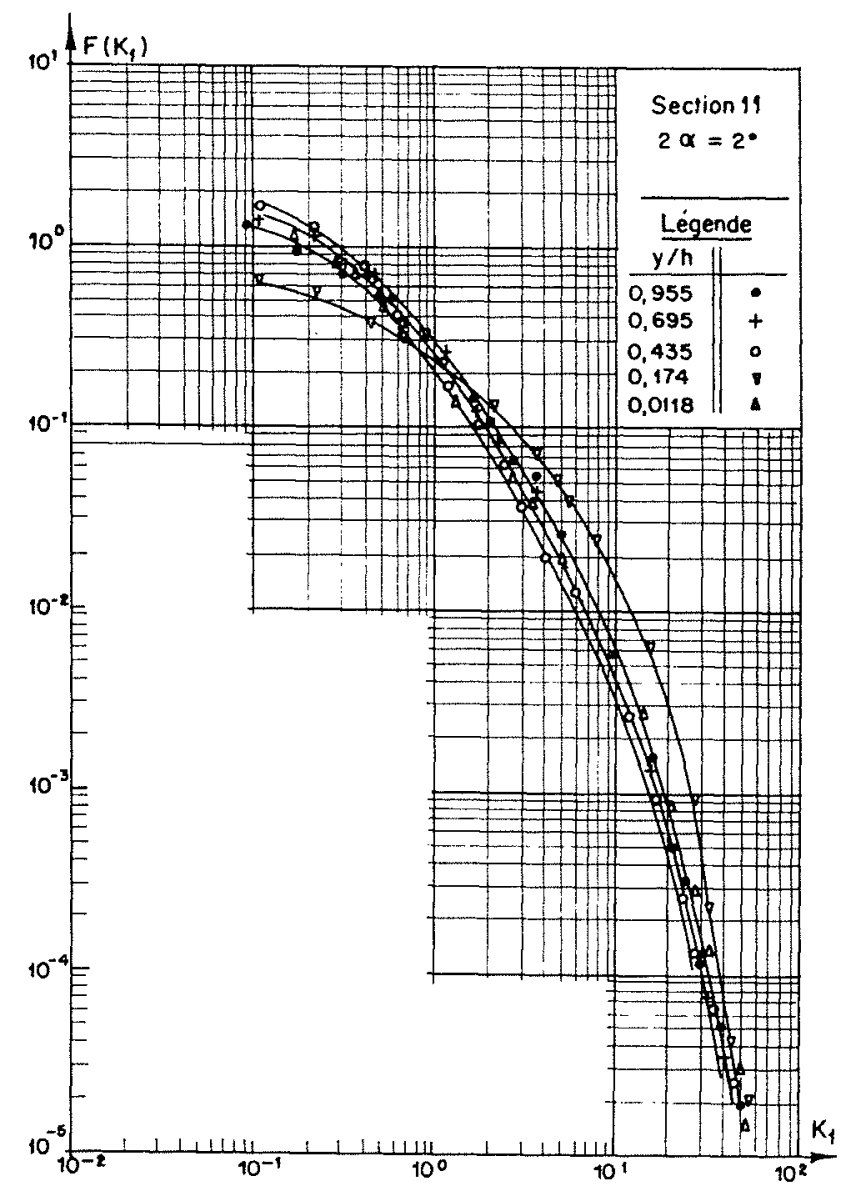

$F_{I G}$ 15. - Fonction spectrale de la fluctuation longitudinale de vitesse; angle $2 \alpha=2^{\circ} ;$ section 11 .

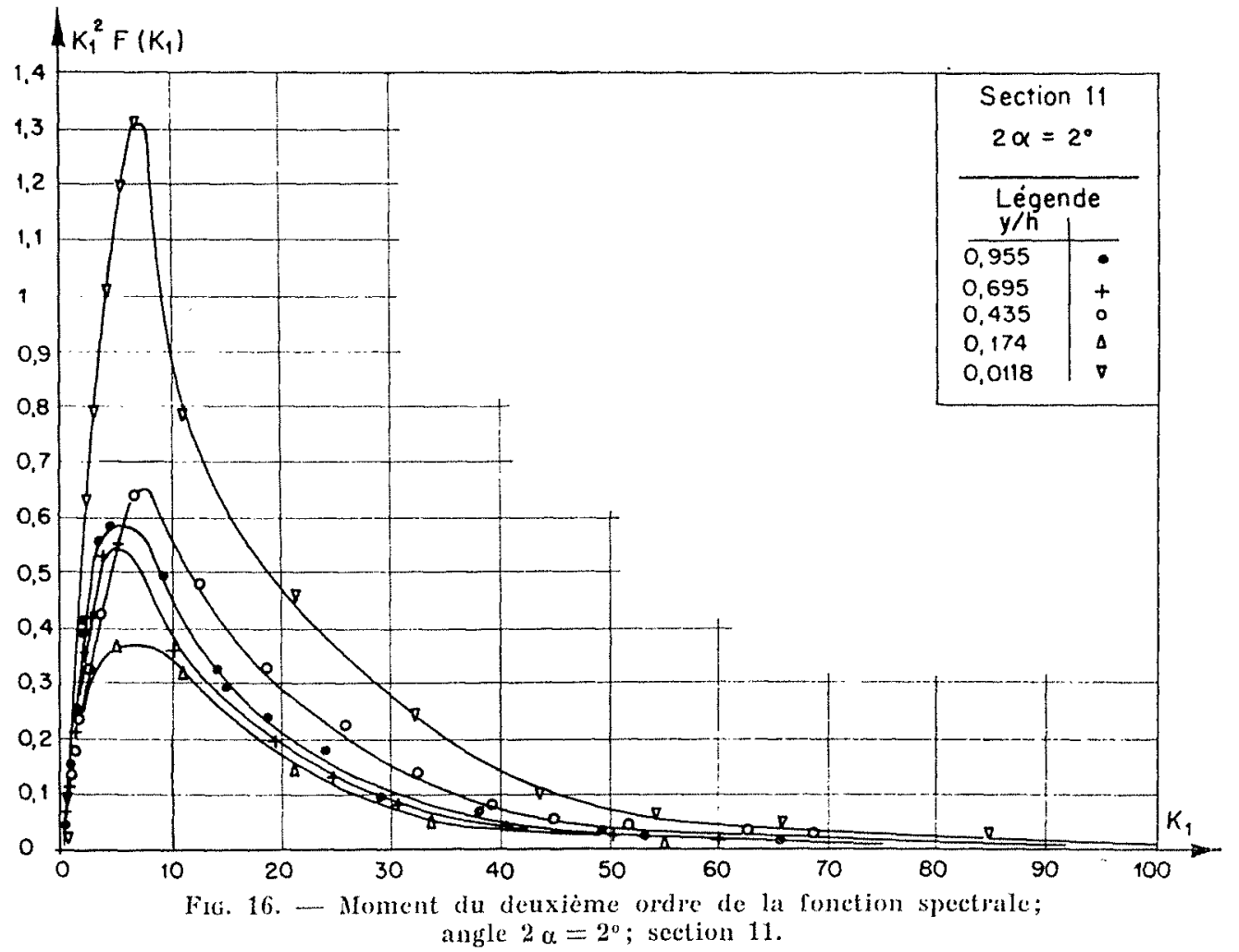




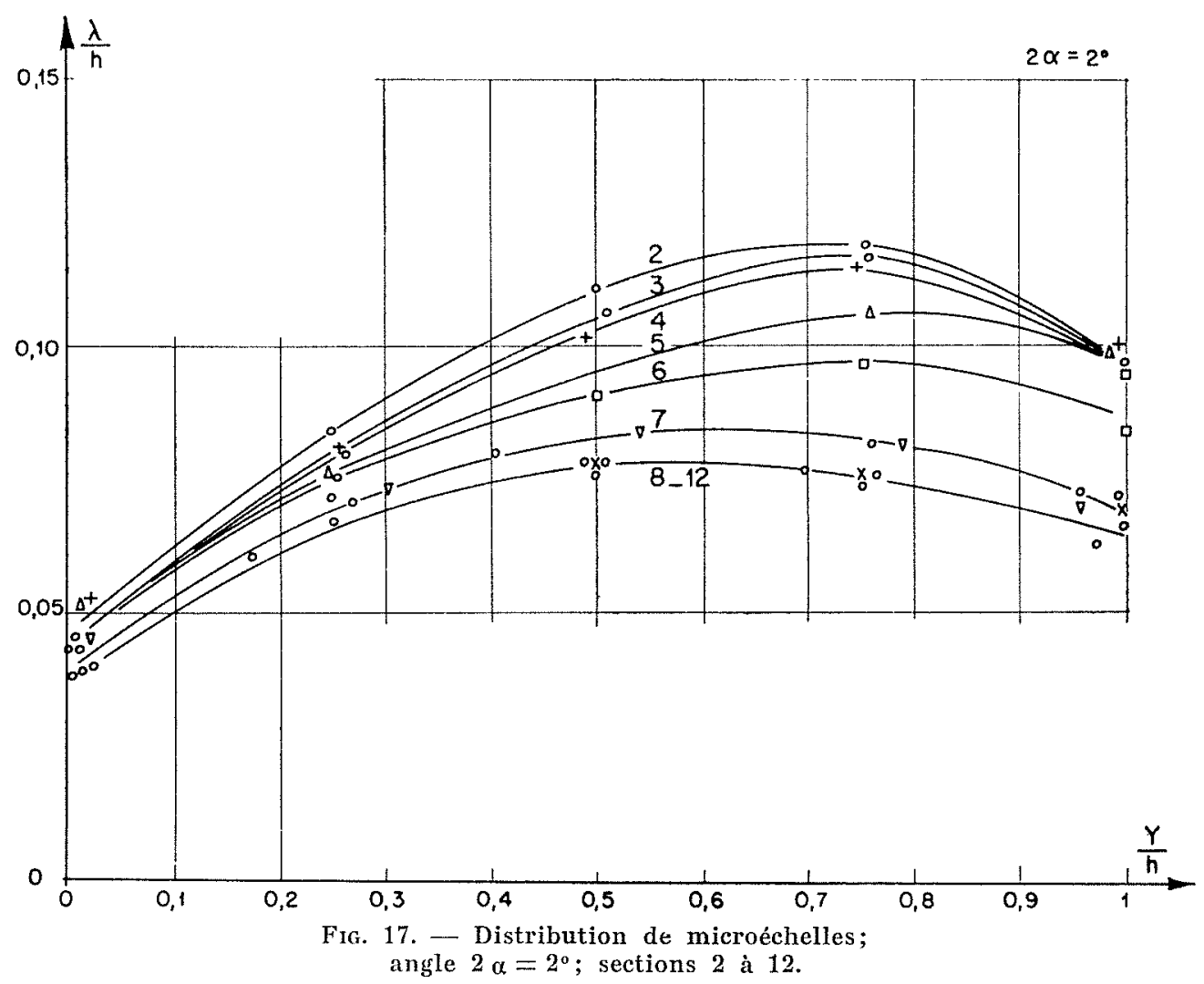

deur des dimensions transversales de l'écoulement; ce résultat, également obtenu par LAUfFer pour un écoulement en canal plan, est une des contradictions apportées à la théorie de la longueur de mélange qui associe la fluctuation turbulente en un point au gradient local de vitesse en ce point.

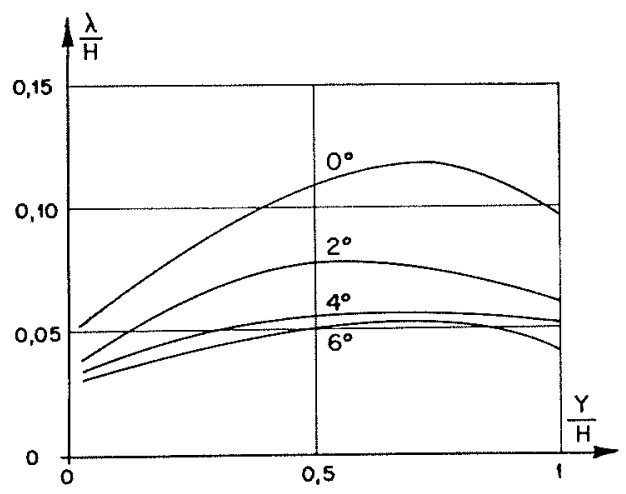

Fig. 18. - Distribution de $\lambda / h$ pour les différents régimes d'équilibre.

\section{Comparaison des Différentes fonctions SPECTRALES.}

Pour comparer entre eux les spectres d'énergie obtenus dans les différentes sections de mesure, il nous a paru intéressant d'utiliser une représentation en coordonnées non dimensionnelles.
Parmi les grandeurs caractéristiques d'un spectre, les principales sont l'aire $\bar{u}^{2}$ et le moment du deuxième ordre $\overline{(\partial u / \partial x)^{2}}$, soit encore $u^{\prime}$ et $\lambda$; si la fonction spectrale dépendait principalement de ces grandeurs, et par ailleurs de

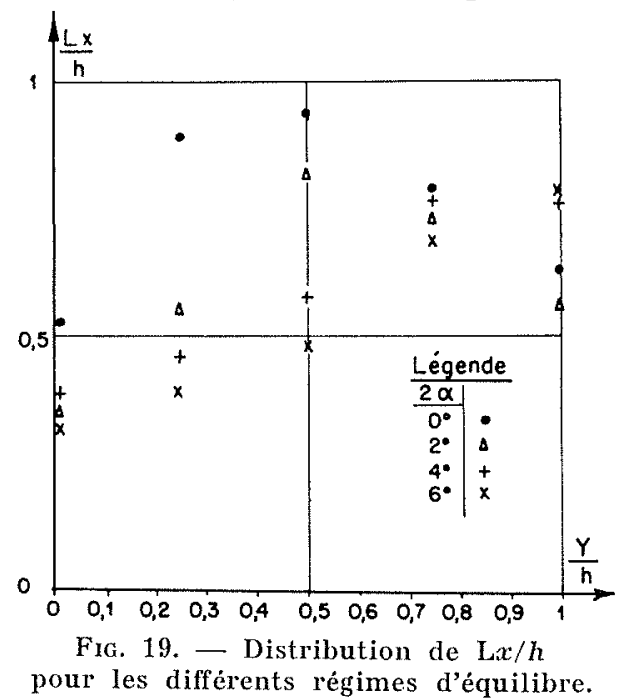

la viscosité cinématique $\vee$ qui caractérise le fluide, l'analyse dimensionnelle montre que l'on peut écrire $F / \lambda=\varphi\left(k_{1} \lambda, u^{\prime} \lambda / y\right)$. On est conduit ainsi naturellement à représenter $F / \lambda$ en fonction de $k_{1} \lambda$, et cette relation est en principe sous la dépendance notamment de $u^{\prime} \lambda / v$ (nombre de 


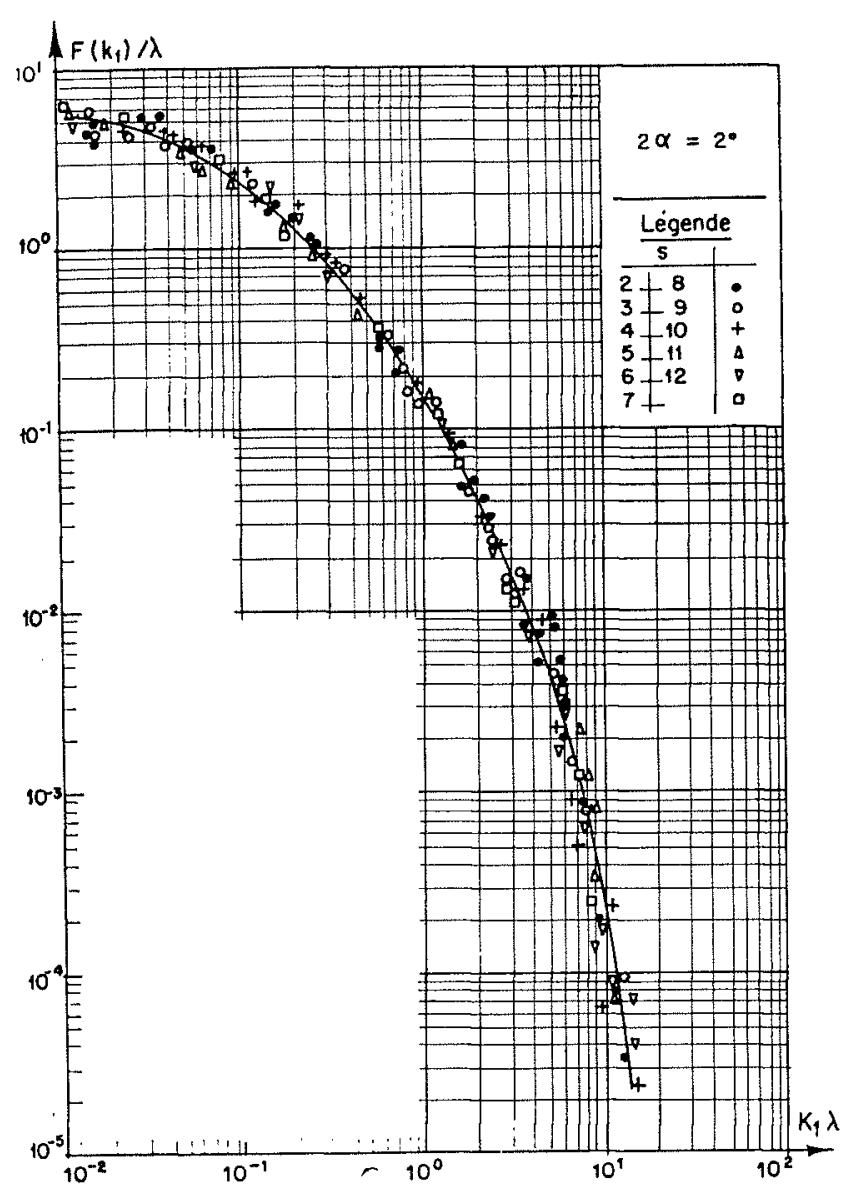

FIG. 20. - Fonction spectrale réduite de la fluctuation longitudinale de vitesse; angle $2 \alpha=2^{\circ} ; y / h=1$.

Reynolds de turbulence) et d'autres paramètres caractérisant, dans notre cas, la non-homogénéité et l'anisotropie de la turbulence.

Nous avons à titre d'exemple tracé dans ces coordonnées réduites les courbes spectrales correspondant au point central $(y / h=1)$ des différentes transversales de mesure pour l'écoulement dans le divergent de 2 degrés (fig. 20 ) ; on constate, dans la gamme de fréquence explorée, un regroupement approché des diverses courbes spectrales autour d'une courbe unique; ce re-

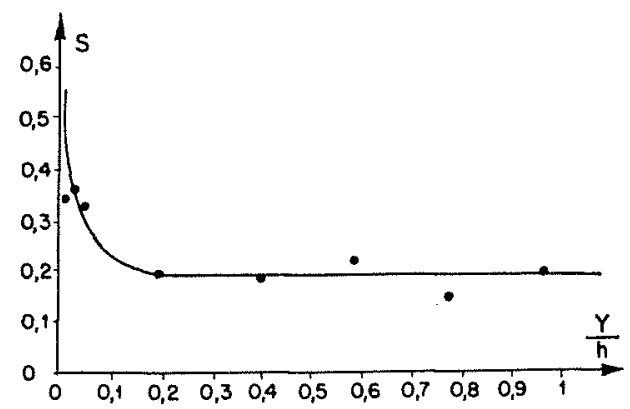

groupement autour de cetle courbe subsiste pour les fonctions spectrales correspondant aux divers points d'une même section de mesure et pour les divers angles de divergence étudiés.

Il convient toutefois de souligner que la similitude obtenue concerne uniquement les spectres des fluctuations longitudinales, la gamme de fréquence explorée, et que le nombre de Reynolds $u^{\prime} \lambda / v$ varie peu dans nos essais; il s'agit donc d'un regroupement approché, plutôt que d'une loi physique, que la complexité des résultats acquis en turbulence isotrope permet difficilement d'escompter.

4. Corrélations TRIPLES. INTERMITTENCE DE L'ÉCOULEMENT TURBULENT AU VOISINAGE DE LA PAROI.

Parmi les autres grandeurs statistiques liées à la turbulence nous avons mesuré les coefficients de dissymétrie $\mathrm{S}$ et d'aplatissement $\mathrm{T}$ des distributions de probabilité des dérivées des fluctuations longitudinales de vitesse, à savoir :

$$
\begin{aligned}
\mathrm{S}=\frac{-\overline{(\partial u / \partial t)^{3}}}{\left[\overline{(\partial u / \partial t)^{2}}\right]^{3 / 2}} \quad \mathrm{~T}=\frac{\overline{(\partial u / \partial t)^{4}}}{\left[\overline{\left.(\partial u / \partial t)^{2}\right]^{2}}\right.} \\
\text { et } \left.\mathrm{T}_{1}=\frac{\overline{\left(\partial^{2} u / \partial t^{2}\right)^{4}}}{\left[\left(\partial^{2} u / \partial t^{2}\right)^{2}\right.}\right]^{2}
\end{aligned}
$$

Les valeurs de ces coefficients, mesurées lors d'un essai dans une des sections de mesure, sont groupées sur la figure 21; on remarque que, sauf au voisinage de la paroi, ces coefficients ont des valeurs constantes (à la dispersion près des mesures qui sont délicates) égales respectivement pour $\mathrm{S}, \mathrm{T}$ et $\mathrm{T}_{1}$ à $0,2,3,7$ et 4,5 .

Le premier résultat de ces mesures est de permettre une comparaison avec des résultats acquis par d'autres chercheurs et destinés à vérifier une conséquence de la théorie de l'isotropie

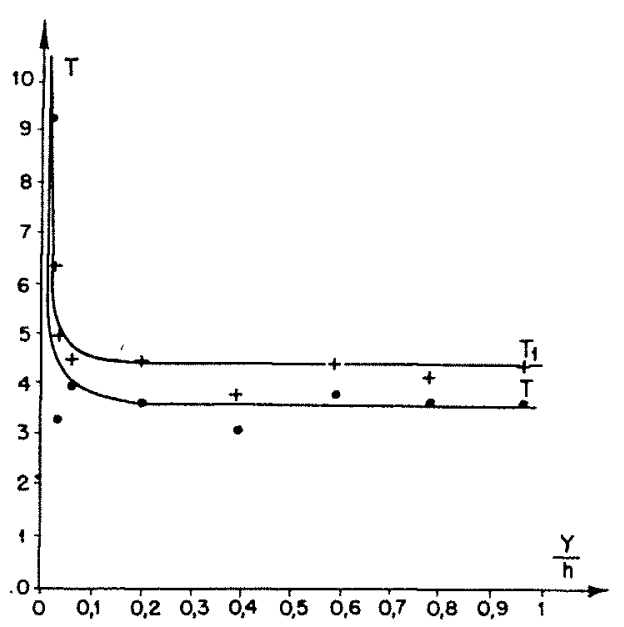

Fig. 21. - Distribution de $S, T, T_{1}$ dans une transversale de mesure. 
locale de Kolmogoroff; si cette théorie était vérifiée, pour des nombres de Reynolds de turbulence assez élevés (supérieurs à 200 par exemple), les valeurs de $S$, $T$ et $T_{1}$ devraient être des constantes universelles; en fait les valeurs que nous avons obtenues sont différentes de celles trouvées par TownSENd et STEWART (18) (par exemple $\mathrm{S}=0,2$, alors que ces auteurs trouvent $0,4)$, ce qui pourrait s'expliquer par la différence des nombres de Reynolds $u^{\prime} \gamma / \gamma$ (dont la valeur est égale à 200 dans nos essais, et varie chez eux de 20 à 60 ); aucune conclusion ne peut donc être valablement tirée de ces résultats, tant que d'autres' mesures concernant des écoulements turbulents de nature différente et des nombres de Reynolds de turbulence élevés n'auront pas été entreprises.

D'autre part, on remarque d'après la figure 21 qu'au voisinage de la paroi les valeurs de $S$, $T$ et $\mathrm{T}_{1}$ varient rapidement; nous avons essayé d'interpréter ces résultats en étendant au film laminaire la notion d'intermittence, appliquée récemment à l'étude de la zone frontière entre une couche limite développée à partir d'une paroi plane et le fluide libre; en un point situé près de la paroi on aurait une succession aléatoire dans le temps entre l'écoulement laminaire et l'écoulement turbulent. En définissant un coefficient d'intermittence $\gamma$ comme la fraction du temps pendant lequel l'écoulement est turbulent, si $e$ est une quantité aléatoire liée à la turbulence, on aura $e=\gamma e^{\prime}, e^{\prime}$ étant la valeur que prendrait $e$ si l'écoulement était toujours turbulent; on aura par exemple :

$$
\left(\frac{\partial u}{\partial t}\right)^{2}=\gamma\left(\overline{\frac{\partial u}{\partial t}}\right)^{2^{\prime}}
$$

etc., d'où l'on déduil facilement que :

$$
\mathrm{S}=\frac{1}{\sqrt{\gamma}} \mathrm{S}^{\prime} \quad \mathrm{T}=\frac{1}{\gamma} \mathrm{T}^{\prime} \quad \mathrm{T} \mathrm{T}_{1}=\frac{1}{\gamma} \mathrm{T}_{1}^{\prime}
$$

Prenant pour valeur de ' $\mathrm{T}^{\prime}$ et $\mathrm{T}^{\prime}{ }_{1}$ les valeurs mesurées dans la zone centrale de la section de mesure $\left(\mathrm{T}^{\prime}=3,7 ; \mathrm{T}_{1}^{\prime}=4,5\right)$, les valeurs de $\gamma$ obtenues à partir des' mesures de $\mathrm{T}$ et $\mathrm{T}_{1}$ près de la paroi sont rassemblées dans le tableau suivant :

\begin{tabular}{|l|c|c|c|l|}
\hline$y / h$ & $\mathrm{~T}$ & $\mathrm{~T}_{1}$ & $\gamma_{\mathrm{T}}$ & $\gamma_{\mathrm{T}_{1}}$ \\
\cline { 1 - 3 } 0,00575 & 9,3 & 10 & 0,4 & 0,45 \\
\cline { 1 - 2 } 0,0077 & 4,8 & $\mathbf{6 , 4}$ & 0,77 & 0,7 \\
0,023 & 3,3 & 5 & 1,1 & 0,9 \\
0,042 & 4,0 & 4,5 & 0,93 & 1 \\
0,196 & 3,7 & 4,5 & 1 & $\mathbf{1}$ \\
\hline
\end{tabular}

L'épaisseur du film laminaire, définie comme la zone dans laquelle la répartition des vitesses est linéaire, étant égale à 0,01 pour la section envisagée ci-dessus, il apparaît que le phénomène d'intermittence s'étend largement de part et d'autre de cette frontière théorique, en particulier dans la zone (《 buffer-layer ») où la répar-

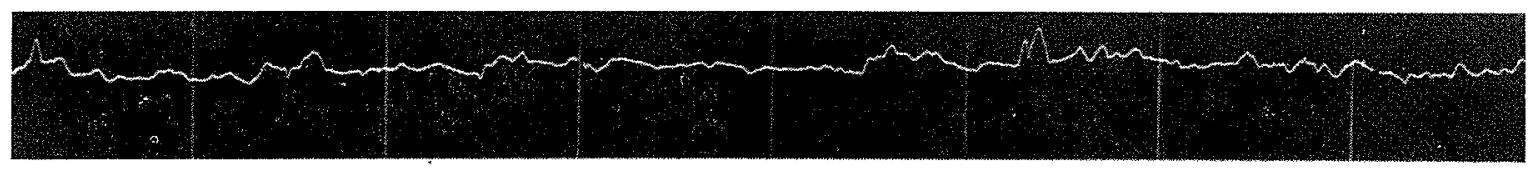

$\left(u_{*} y / v\right)=6$

$u^{\prime}=0,6 \mathrm{~m} / \mathrm{s}$

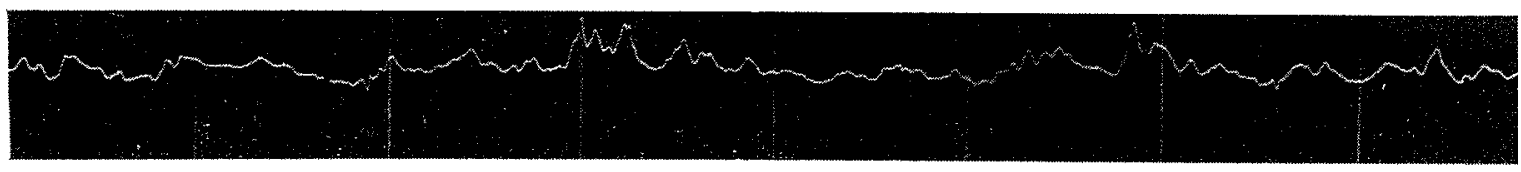

$\left(u_{*} y / v\right)=8$

$u^{\prime}=0,96 \mathrm{~m} / \mathrm{s}$

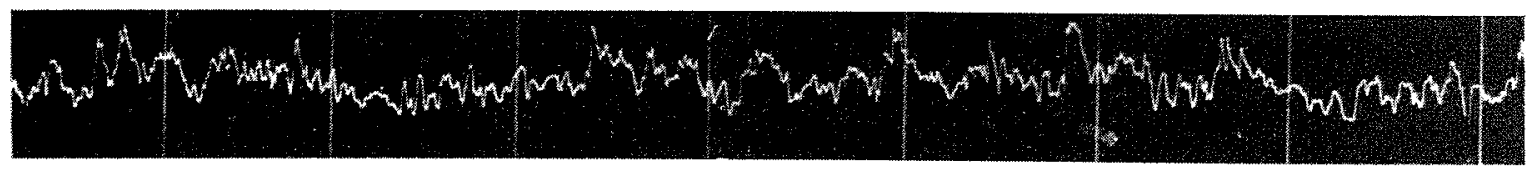

$\left(u_{*} y / v\right)=23$

$u u^{\prime}=1,49 \mathrm{~m} / \mathrm{s}$
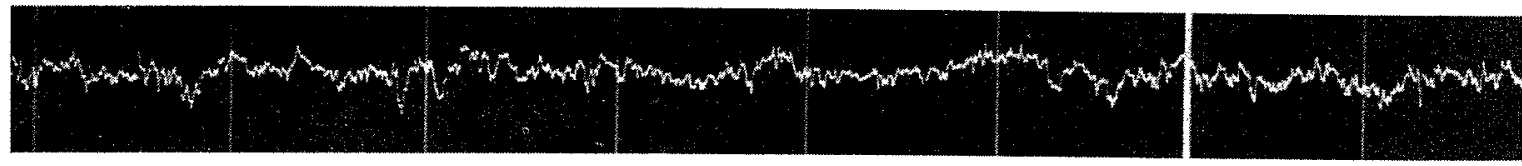

$\left(u_{*} y / v\right)=1.000$

$u^{\prime}=1,1 \mathrm{~m} / \mathrm{s}$

Fră. 22. - Oscillogrammes des fluctuations longitudinales de vitesse $u$. 
tition des vitesses moyennes n'est pas encore logarithmique.

Des oscillogrammes des fluctuations $\boldsymbol{u}$ ont été enregistrés dans la section envisagée ci-dessus (fig. 22) et confirment qualitativement le phénomène d'intermittence; pour les points les plus près de la paroi $\left[\left(u_{*} y / v\right)=6\right.$ et 8$]$, il semble que l'on ait des «bouffées de turbulence» faisant suite à des périodes plus calmes; bien que ces enregistrements ne permettent pas une détermination précise du facteur d'intermittence, les valeurs' obtenues à partir des mesures de $T$ et $T_{1}$ sont toutefois en bon accord avec ceux-ci.

Les résultats présentés dans ce dernier paragraphe n'ont été acquis que par un petit nombre de mesures; nous ne les présentons donc que sous réserve de vérifications systématiques ultérieures et, en particulier, en ce qui concerne l'intermittence au voisinage de la paroi, de mesures dans un tunnel de dimensions plus grandes permettant d'explorer plus facilement le film laminaire.

\section{BIBLIOGRAPHIE SOMMAIRE}

(1) Batchelon (G. K.). - Note on free turbulent flows, with special reference to the two-dimensional wake.

Journ. of Aeronautical Science, juillet 1955.

(2) TAYLOR (G. I.). - Statistical theory of turbulence. Parts 1-4; Proc. Roy. Soc., London 1935 and part $5,1936$.

(3) Karman (T.) and Howanth (L.). - On the statistical theory of isotropic turbulence. Proc. Roy. Soc., London, 1937.

(4) Townsend (A.A.). - The structure of the turbulent boundary layer. Proc. Camb. Phil. Soc., vol. 47, 1951.

(5) Klebanoff (P.S.) and DieHL (T.W.). - Some features of artificially thickened fully developed turbulent boundary layers with zero pressure gradient. N.A.C.A. Techn., note $2475,1951$.

(6) Schubatun (G. B.) and Klebanoff (P.S.). - Investigation of separation of the turbulent boundary layer.

N.A.C.A. Techn., note 2133, 1950.

(7) Clauser (F. H.). - Turbulent boundary layers in adverse pressure gradients. Journ. of Aero. Science, vol. 21, 1954.

(8) Connsin (S.). - Investigation of flow in an axially symmetrical heated jet of air. N.A.C.A. Wartime, report W.94, 1943.

(9) Townsend (A.A.). - The fully developed turbulent wake of a circular cylinder.
Australian Journ. Sci. Res., ser. A, vol. 2, $\mathrm{n}^{\circ} 4$, 1949.

(10) LAUFER (J.). -- Investigation of a turbulent flow in a two-dimensional channel. N.A.C.A. Tech., note 2123, 1950.

(11) Laufer (J.). - The structure of turbulence in fully developed pipe flow. N.A.C.A. Tech., note 2954, 1953.

(12) Nikunadze (J.). - Untersuchungen über die Strömungen des Wassers in konvergentens und divergenten Kanälen.

Forsch-Arb. Geb. Ing-Wes., Heft 289, 1929

(13) Young (A.D.) and Mass (J.N.). - The behavior of a pitot-tube in a traverse pressure gradient. Aero. Res. Council, Rep. 1770, 1936.

(14) Frenkied (F.N.). - Effects of wire-length in turbulence investigations with a hot-wire anemometer. The Aeronautical Quartely, vol. V, part 1, 1954.

(15) Cooper (R.). - Turbulence measurement with the hot-wire anemometer. Agardograph 14, NATO, 1955.

(16) Goldstein (S.). - Modern developments in fluid mechanics. Oxford University Press, 1938.

(17) Taylon (G. I.). - The spectrum of turbulence. Proc. Roy. Soc., London, 1938.

(18) Batchelon (G. K.). - The theory of homogeneous turbulence. Cambridge University Press, 1953 (p. 118).

\section{DISCUSSION}

Président : M. BergenoN

M. le Président croit qu'il exprime l'opinion de tout le monde en félicitant vivement M. MiLliat pour son travail très important et pour la manière dont il l'a présenté : il a montré le phénomène de la turbulence dans un cas très particulier et très intéressant, celui de la divergence. Mis en évidence par les méthodes expérimentales, ces phénomènes apparaissent ainsi sous une forme concrète qui s'oppose à l'abstraction des méthodes statistiques. Les résultats obtenus apportent une contribution intéressante à l'ensemble des travaux poursuivis sur le comportement du phénomène de la turbulence.
M. le Président demande à M. Muliat s'il n'y aurait pas intérêt à poursuivre ces travaux avec des angles de divergence s'accentuant encore un peu.

M. Muliat répond que si l'on prend des angles de divergence plus grands, 8 ou $10^{\circ}$ par exemple, on sera gêné par le phénomène de décollement.

Sur Ia demande de M. Outrey, M. Mr. at indique les dimensions globales du tunnel : longueur totale : $4 \mathrm{~m}$; hauteur : $80 \mathrm{~cm}$.

M. le Président remercie très vivement M. Muratat. 